\title{
A facile one-pot method for the synthesis of polysubstituted phthalides derivatives
}

Xiu-Yan Yang ${ }^{1,2, ~ *, ~ X i a o-Q i a n ~} \mathrm{Xu}^{1,2,{ }^{*}, \text { Xiao-Kui Wang }}{ }^{2}$, Zhi-Bing Zheng ${ }^{2}$, Guo-Ming $\mathrm{Zhao}^{2 \S}$, Song $\mathrm{Li}^{2 \S}$

${ }^{1}$ Shenyang Pharmaceutical University, Shenyang 110016, PR China

${ }^{2}$ Institute of Pharmacology and Toxicology, Academy of Military Medical Sciences, Beijing 100850, PR China

Supplementary Information

Section A: General Information

Section B: Experimental procedures and spectral analysis

Section C: ${ }^{1} \mathrm{H},{ }^{13} \mathrm{C}$ NMR, IR Spectra and element analysis of compounds $1 \mathrm{a}-1 \mathrm{j}$ 


\section{GENERAL}

${ }^{1} \mathrm{H}$ NMR and ${ }^{13} \mathrm{C}$ NMR spectra were recorded at $400 \mathrm{MHz}$ and $100 \mathrm{MHz}$ on JNM-ECA-400 instrument with tetramethylsilane as an internal standard in the

DMSO. IR spectra were recorded in KBr disk using a Nicolet 6700 FT-IR spectrophotometer. ESI-MS (high resolution) mass spectra were obtained by using a Waters Xevo G2 Qtof (ESI) mass spectrometer. Melting points were determined using a RY-1 apparatus and were uncorrected. 
Typical experimental procedure: Ethyl 4-chloroacetoacetate (2 mmol) and ethanal (1 mmol) were placed in a flask under an atmosphere of nitrogen. Piperidine (1 mmol), glacial acetic acid $(1 \mathrm{mmol})$, indion $(1 \mathrm{mmol})$ concentrated hydrochloric acid $(0.01$ $\mathrm{ml})$ and ethanol $(10 \mathrm{ml})$ was added. The resulting mixture was heated at $80{ }^{\circ} \mathrm{C}$ for 12 h. After cooling to room temperature, the solvent was removed by evaporation. The residue was poured into $50 \mathrm{ml}$ of water and extracted with ether. The organic layer was separated and was washed with saturated sodium chloride solution. After dried over sodium sulfate, the solvent was removedand the residue was purified by column chromatography on silica gel to give the product $1 \mathrm{a}(0.15 \mathrm{~g}$, white powder) in $63 \%$ yield.

\section{Selected spectral data of new compounds :}

Compound 1a: mp: $138.3-139.1^{\circ} \mathrm{C} ;{ }^{1} \mathrm{H}$ NMR (DMSO-d6, $\left.400 \mathrm{MHz}\right): \delta$, ppm, $11.12(\mathrm{~s}$, 1H, OH), 6.91(s, 1H, Ph-H), 5.24(s, 2H, CH2), 4.3(q, 2H, CH2), 2.45(s, 3H, CH3), 1.29(t, 3H, CH3); ${ }^{13} \mathrm{C}$ NMR (DMSO-d6, $\left.100 \mathrm{MHz}\right) \delta$, ppm, 170.2, 166.7 , 159.5, 150.9, 136.9, 124.2, 123.6, 113.3, 106.6, 68.3, 61.0, 14.0, 13.6; HRMS: cal. For $\mathrm{C}_{12}$ $\mathrm{H}_{11} \mathrm{O}_{5}$ 235.0612; found 235.0612.

Compound 1b: mp:162.5-163.2 ${ }^{\circ} \mathrm{C} ;{ }^{1} \mathrm{H}$ NMR (DMSO-d6, $\left.400 \mathrm{MHz}\right): \delta$, ppm, 11.12(s, 1H, OH), 6.91(s, 1H, Ph-H), 5.24(s, 2H, CH2), 4.3(q, 2H, CH2), 2.81 (t, 2H, CH2), 1.53 (m, 2H, CH2), 1.29 (t, 3H, CH3), 0.90(t, 3H, CH3); 13C NMR (DMSO-d6, 100 MHz) $\delta$, ppm, 169.8, $166.7,159.5,151.2,141.5,123.9,112.9,106.6,68.3,60.9,30.0$, 42.0, 14.0,14.0; MS (FAB), HRMS: cal. For $\mathrm{C}_{14} \mathrm{H}_{15} \mathrm{O}_{5}$ 263.0925; found 263.0920. 
Compound 1c: mp: $181.5-181.8^{\circ} \mathrm{C} ;{ }^{1} \mathrm{H}$ NMR (DMSO-d6, $400 \mathrm{MHz}$ ): $\delta$, ppm, 11.43 (s, 1H, OH), 7.65 (d, 1H, Ph-H), 7.39 (m, 2H, Ph-H), 7.15 (m, 2H, Ph-H), 5.34(m, 2H, CH2), 3.89 (q, 2H, CH2), 0.79(t, 3H, CH3); IR (KBr): v 3343; 1750; 1675; 1588; $1434 \mathrm{~cm}^{-1}$; m/e 379.0(M + 1) ; Anal: cal. For $\mathrm{C}_{17} \mathrm{H}_{13} \mathrm{BrO}_{5}$ : C, 54.13; H, 3.47; Found: C, 54.13; H, 3.56.

Compound 1d: mp: 230.3-230.8 ${ }^{\circ} \mathrm{C},{ }^{1} \mathrm{H}$ NMR (DMSO-d6, $400 \mathrm{MHz}$ ): $\delta$, ppm, 11.38 (s, 1H, OH), 7.46 (d, 2H, Ph-H), 7.26 (d, 2H, Ph-H), 7.11 (s, 1H, Ph-H), 5.29(s, 2H, CH2), 3.96(q, 2H, CH2), 0.90(t, 3H, CH3); IR (KBr): v 3497; 1755; 1672; 1588; $1434 \mathrm{~cm}^{-1}$; m/e 333.1(M + 1); Anal: cal. For $\mathrm{C}_{17} \mathrm{H}_{13} \mathrm{ClO}_{5}$ : C, 61.36; H, 3.94; Found: C, 61.38; H, 3.98.

Compound 1e: mp: $172.1-172.5^{\circ} \mathrm{C} ;{ }^{1} \mathrm{H}$ NMR (DMSO-d6, $\left.400 \mathrm{MHz}\right): \delta, \mathrm{ppm}, 11.16(\mathrm{~s}$, 1H, OH), 7.35 (m, 1H, Ph-H), 7.05(m, 2H, Ph-H),6.98(m, 2H, Ph-H), 5.29(m, 2H, CH2), 3.90(q,2H,CH2),3.62 (s,3H,CH3), 0.90(t, 3H, CH3); $\quad$ IR (KBr): v 3497; 1756; 1663; 1597; $1498 \mathrm{~cm}^{-1} ; \mathrm{m} / \mathrm{e} 329.1(\mathrm{M}+1)$; Anal: cal. For $\mathrm{C}_{18} \mathrm{H}_{16} \mathrm{O}_{6}$ : C, 65.85; $\mathrm{H}$, 4.91; Found: C, 65.55; H, 4.95.

Compound 1f: mp: 226.3-226.9 ${ }^{\circ}$; ${ }^{1} \mathrm{H}$ NMR (DMSO-d6, $400 \mathrm{MHz}$ ): $\delta$, ppm, 11.32(s, 1H, OH), 10.48(s, 1H, OH), 7.33(s, 1H, Ph-H), 7.06(m, 2H, Ph-H),6.96(m, 1H, $\mathrm{Ph}-\mathrm{H})$, 5.26(s, 2H, CH2), 4.01(q,2H,CH2), 0.97(t, 3H, CH3); IR (KBr): v 3343; 1741; $1664 ; 1604 ; 1510 \mathrm{~cm}^{-1} ; \mathrm{m} / \mathrm{e} 392.9(\mathrm{M}+1)$; Anal: cal. For $\mathrm{C}_{17} \mathrm{H}_{13} \mathrm{BrO}_{6}: \mathrm{C}$, 51.93; H, 3.33; Found: C, 52.01; H, 3.38. 
Compound 1g: mp: 210.2-210.6 ${ }^{\circ} \mathrm{C} ;{ }^{1} \mathrm{H}$ NMR (DMSO-d6, $\left.400 \mathrm{MHz}\right): \delta, \mathrm{ppm}, 11.22(\mathrm{~s}$, 1H, OH), 9.17(s, 1H, OH), 7.03(s, 1H, Ph-H), 6.81(m, 2H, Ph-H),6.75(m, 1H, Ph-H), 5.26(s, 2H, CH2), 4.01(q,2H,CH2), $3.71(\mathrm{~s}, 3 \mathrm{H}, \mathrm{CH} 3), 0.94(\mathrm{t}, 3 \mathrm{H}, \mathrm{CH} 3) ; \quad$ IR (KBr): v 3448; 1755; 1658; 1597; $1518 \mathrm{~cm}^{-1} ; \mathrm{m} / \mathrm{e} 345.2(\mathrm{M}+1)$; Anal: cal. For $\mathrm{C}_{18} \mathrm{H}$ ${ }_{16} \mathrm{O}_{7}: \mathrm{C}, 62.79 ; \mathrm{H}, 4.68$; Found: $\mathrm{C}, 62.42 ; \mathrm{H}, 4.71$.

Compound 1h: mp: 215.6-216.6 ${ }^{\circ} \mathrm{C} ;{ }^{1} \mathrm{H}$ NMR (DMSO-d6, $\left.400 \mathrm{MHz}\right): \delta, \mathrm{ppm}, 11.33(\mathrm{~s}$, 1H, OH), $9.70(\mathrm{~s}, 1 \mathrm{H}, \mathrm{OH}), \quad$ 7.07(s, 1H, Ph-H), 6.93(s, 1H, Ph-H),6.88(s, 1H, Ph-H), 5.26(s, 2H, CH2), 4.05(q,2H,CH2), $3.78(\mathrm{~s}, 3 \mathrm{H}, \mathrm{CH} 3), 0.99(\mathrm{t}, 3 \mathrm{H}, \mathrm{CH} 3) ; \quad \mathrm{IR}(\mathrm{KBr}): \mathrm{v}$ $3461 ; 1758 ; 1654 ; 1590 ; 1505 \mathrm{~cm}^{-1} ; \mathrm{m} / \mathrm{e} 424.9(\mathrm{M}+1)$; Anal: cal. For $\mathrm{C}_{17} \mathrm{H}_{15} \mathrm{BrO}_{7}$ : C, 51.08; H, 3.57; Found: C, 51.16; H, 3.62.

Compound 1i: mp: 170.4-171.2 ${ }^{\circ} \mathrm{C} ;{ }^{1} \mathrm{H}$ NMR (DMSO-d6, $400 \mathrm{MHz}$ ): $\delta, \mathrm{ppm}, 11.47$ (s, 1H, OH), 7.55 (m, 1H, Ph-H), 7.26 (m, 1H, Ph-H), 7.24 (m, 1H, Ph-H), 7.16 (s, 1H, Ph-H), 5.34 (s, 2H, CH2), 3.95 (q, J = 7 Hz, 2H, CH2), 0.86 (t, J = 7 Hz, 3H, CH3); IR (KBr): v 3344; 1755; 1676; 1604; $1498 \mathrm{~cm}^{-1}$; MS (FAB), m/e $351.2(\mathrm{M}+1)$; HRMS: cal. For $\mathrm{C}_{17} \mathrm{H}_{13} \mathrm{ClFO}_{5} 351.0430$; found 351.0409 .

Compound 1j: mp: 216.9-217.3 ${ }^{\circ} \mathrm{C} ;{ }^{1} \mathrm{H}$ NMR (DMSO-d6, $\left.400 \mathrm{MHz}\right): \delta$, ppm, 11.52 (s, 1H, OH), 8.31 (d, 1H, Ph-H), 8.11 (s, 1H, Ph-H), 7.75 (m, 2H, Ph-H), 7.18 (s, 1H, $\mathrm{Ph}-\mathrm{H})$, 5.32(s, 2H, CH2), 3.97 (q, 2H, CH2), 0.88 (t, 3H, CH3); IR (KBr): v 3496; 1766; 1662; 1530; $1429 \mathrm{~cm}^{-1} ; \quad \mathrm{m} / \mathrm{e} 343.1(\mathrm{M}+1)$; Anal: cal. For $\mathrm{C}_{17} \mathrm{H}_{13} \mathrm{NO}_{7}$ : $\mathrm{C}$, 59.48; H, 3.82; Found: C, 59.56; H, 3.92. 
Compound 1a:

${ }^{1} \mathrm{H}$ NMR
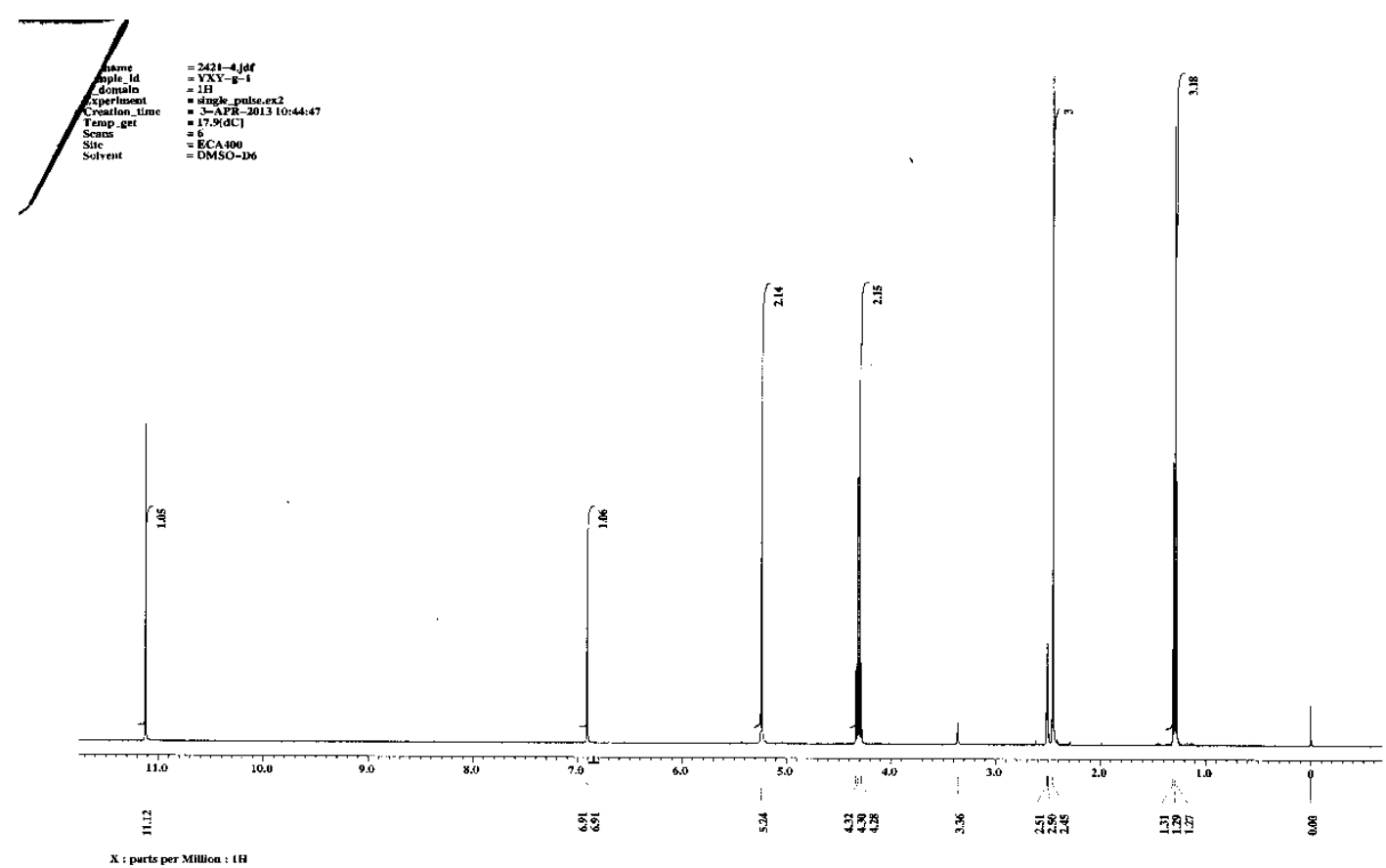

${ }^{13} \mathrm{C}$ NMR 


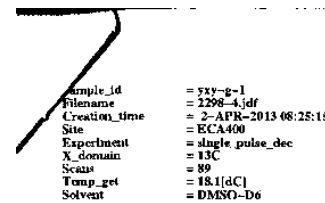

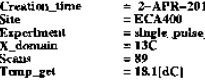

$=\mathrm{D} M \mathrm{MSOD}-\mathrm{D}$ e

HRMS

Mass Spectrum Molecular Formula Report

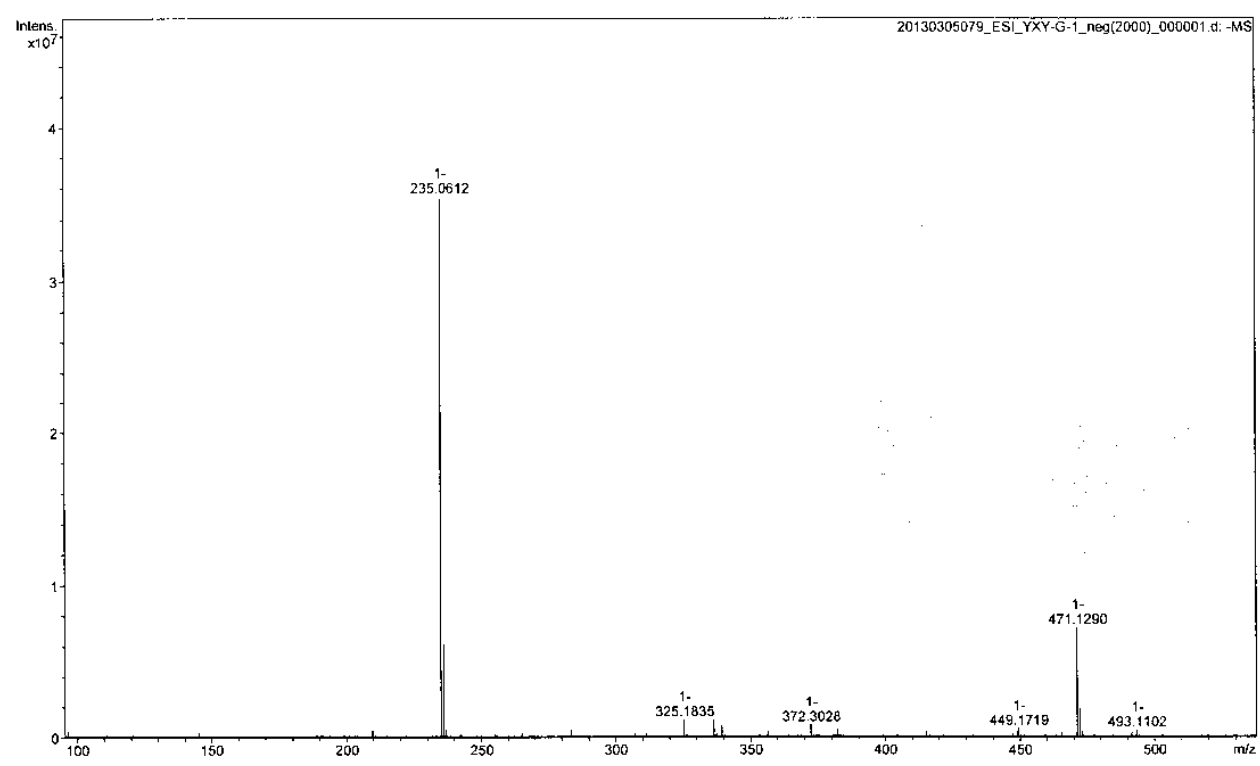

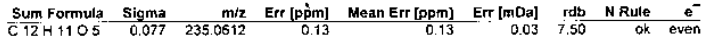


Compound $1 \mathrm{~b}$

${ }^{1} \mathrm{H}$ NMR

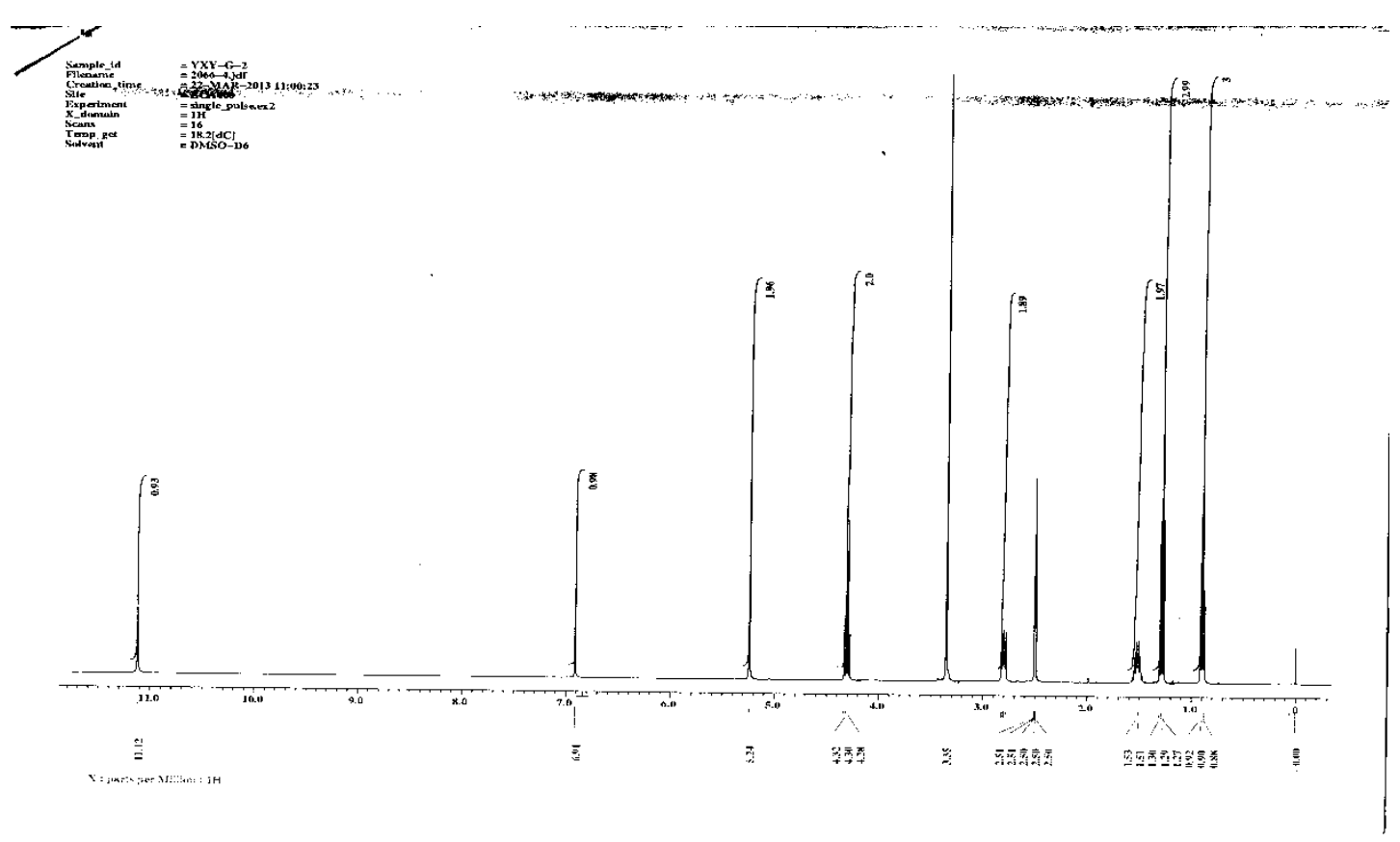

${ }^{13} \mathrm{C}$ NMR
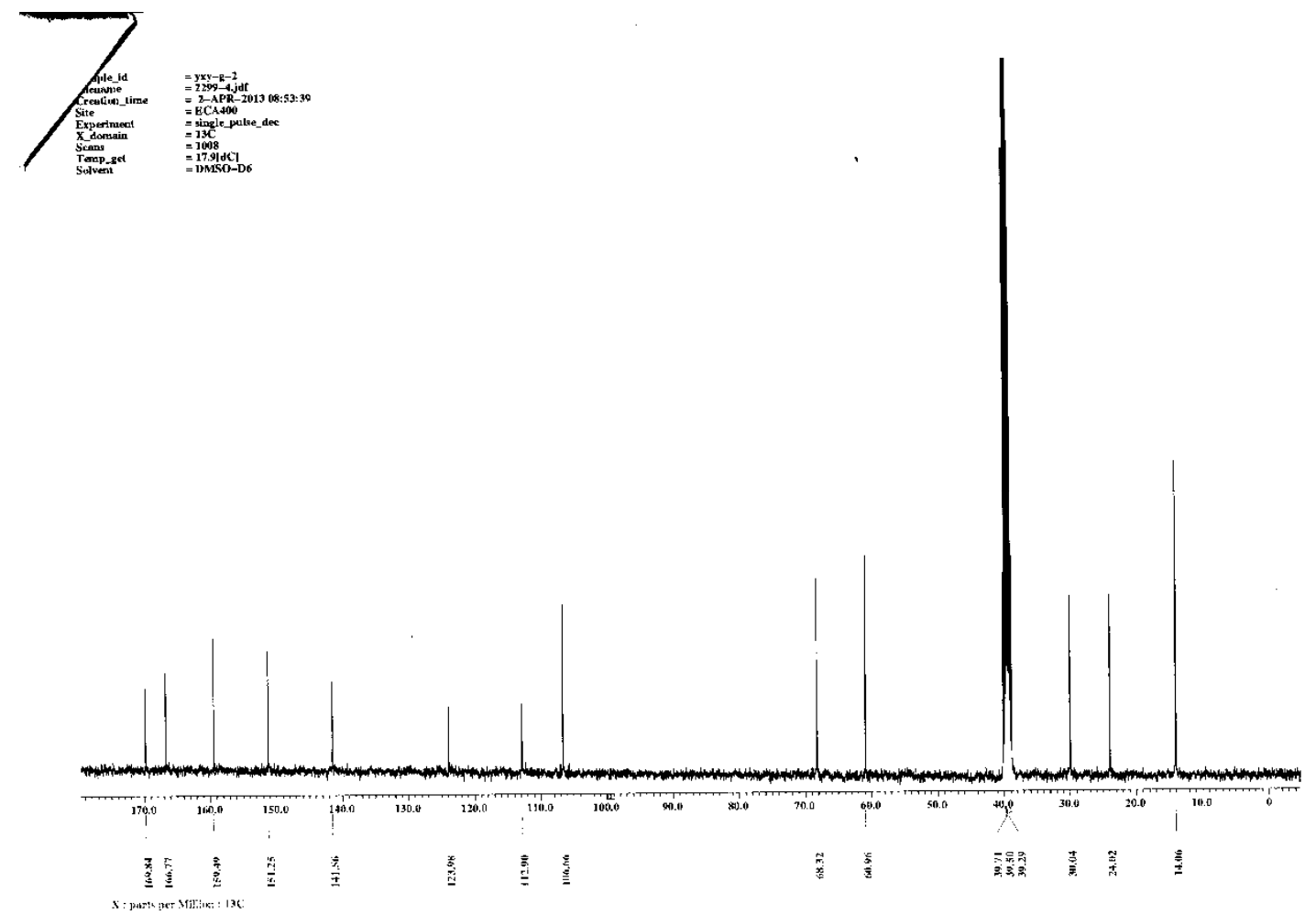


\section{HRMS}

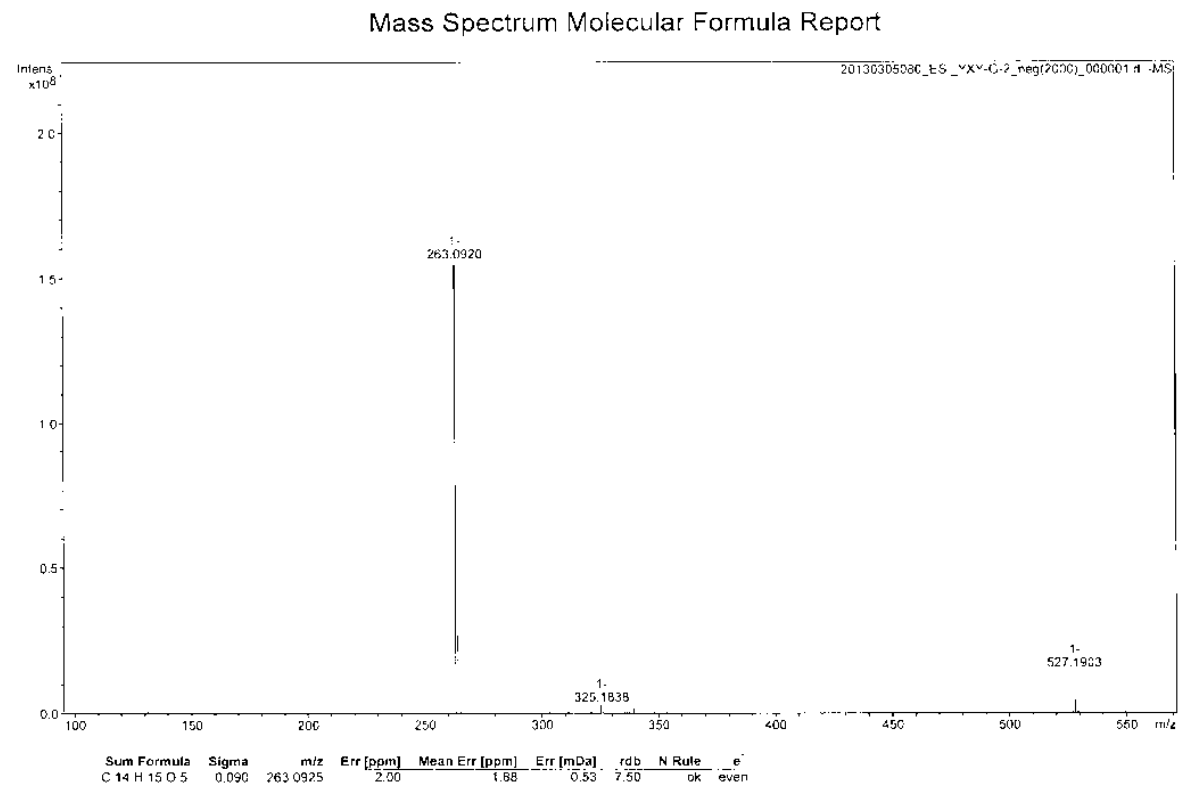

Compound 1c

${ }^{1} \mathrm{H}$ NMR 


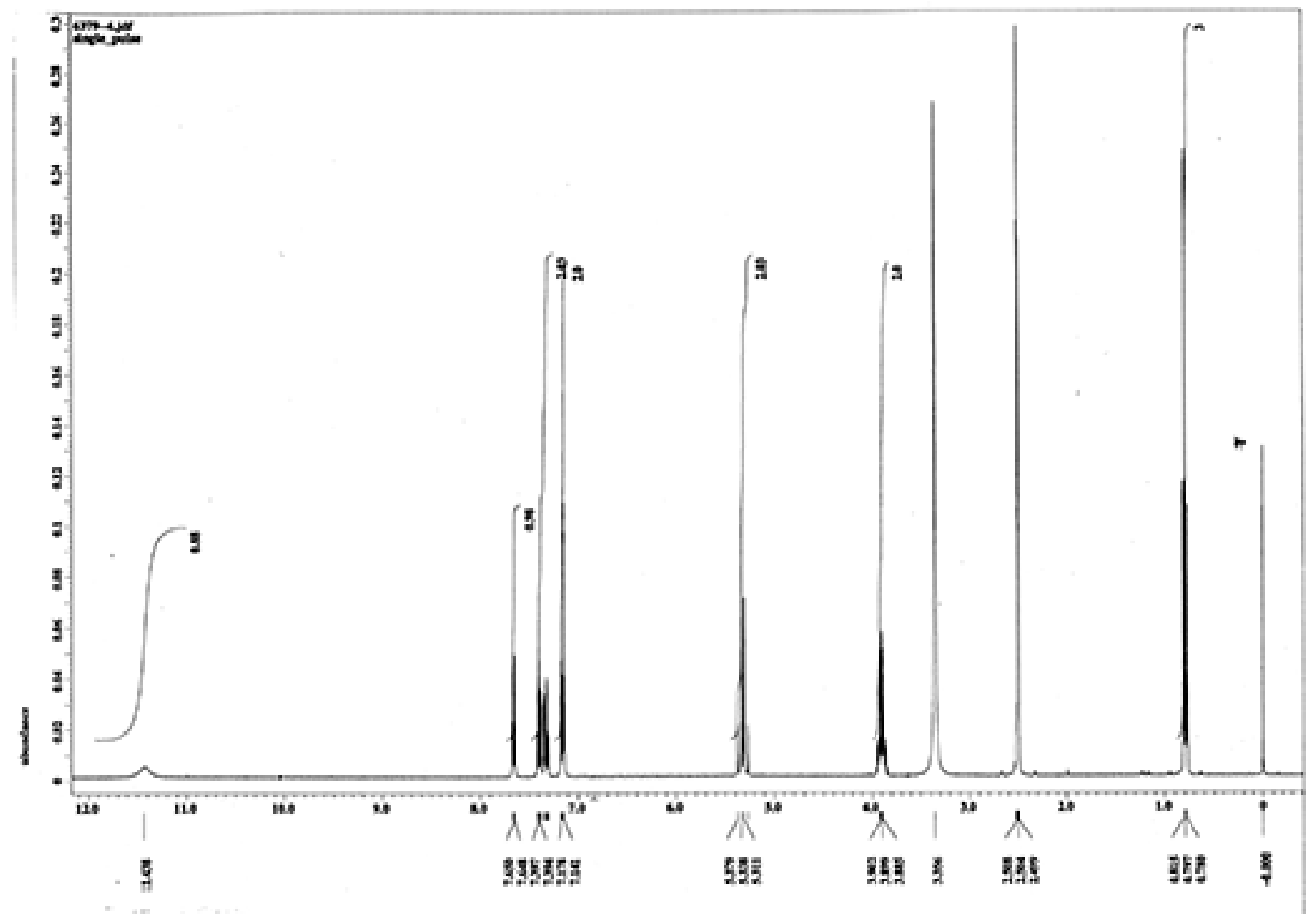

IR

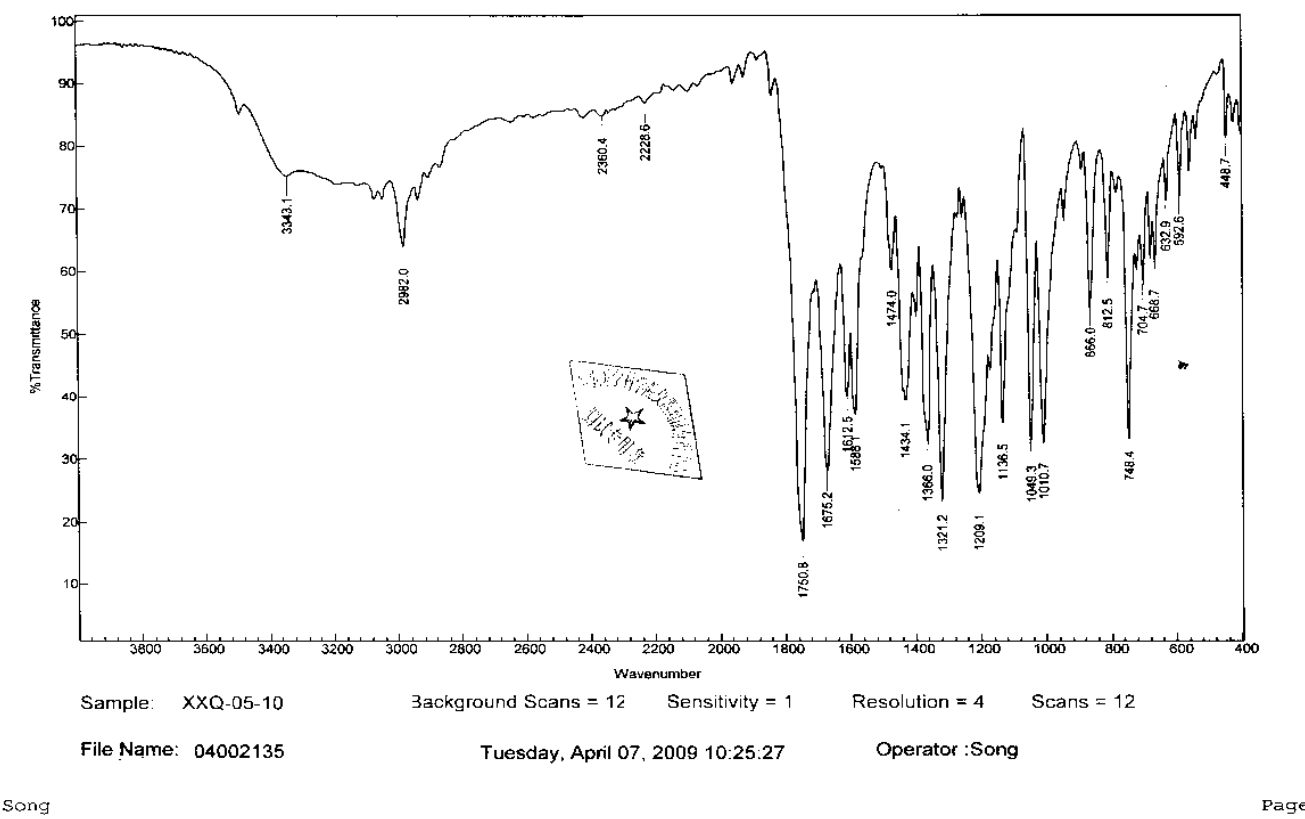




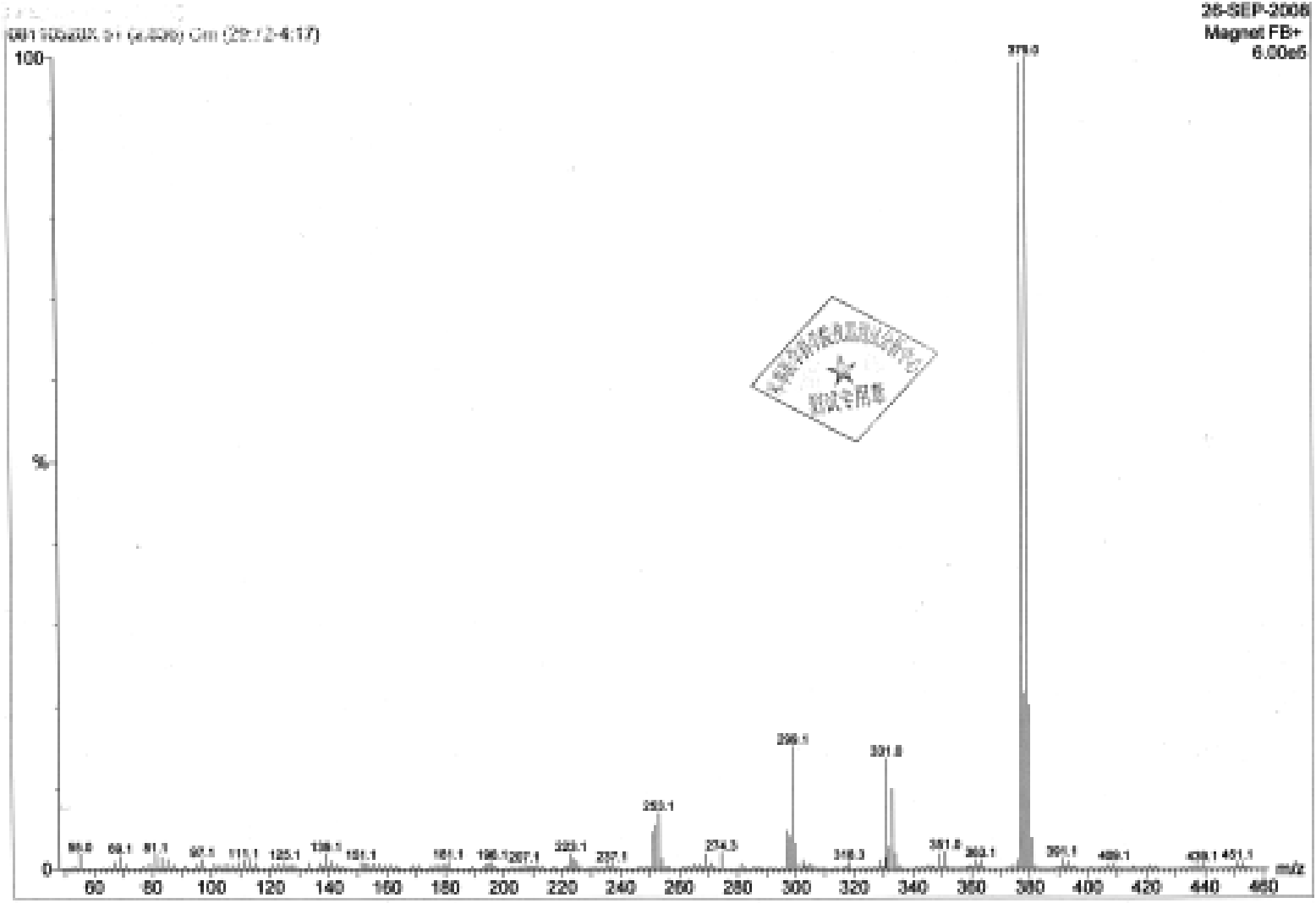

Compound 1d

${ }^{1} \mathrm{H}$ NMR 


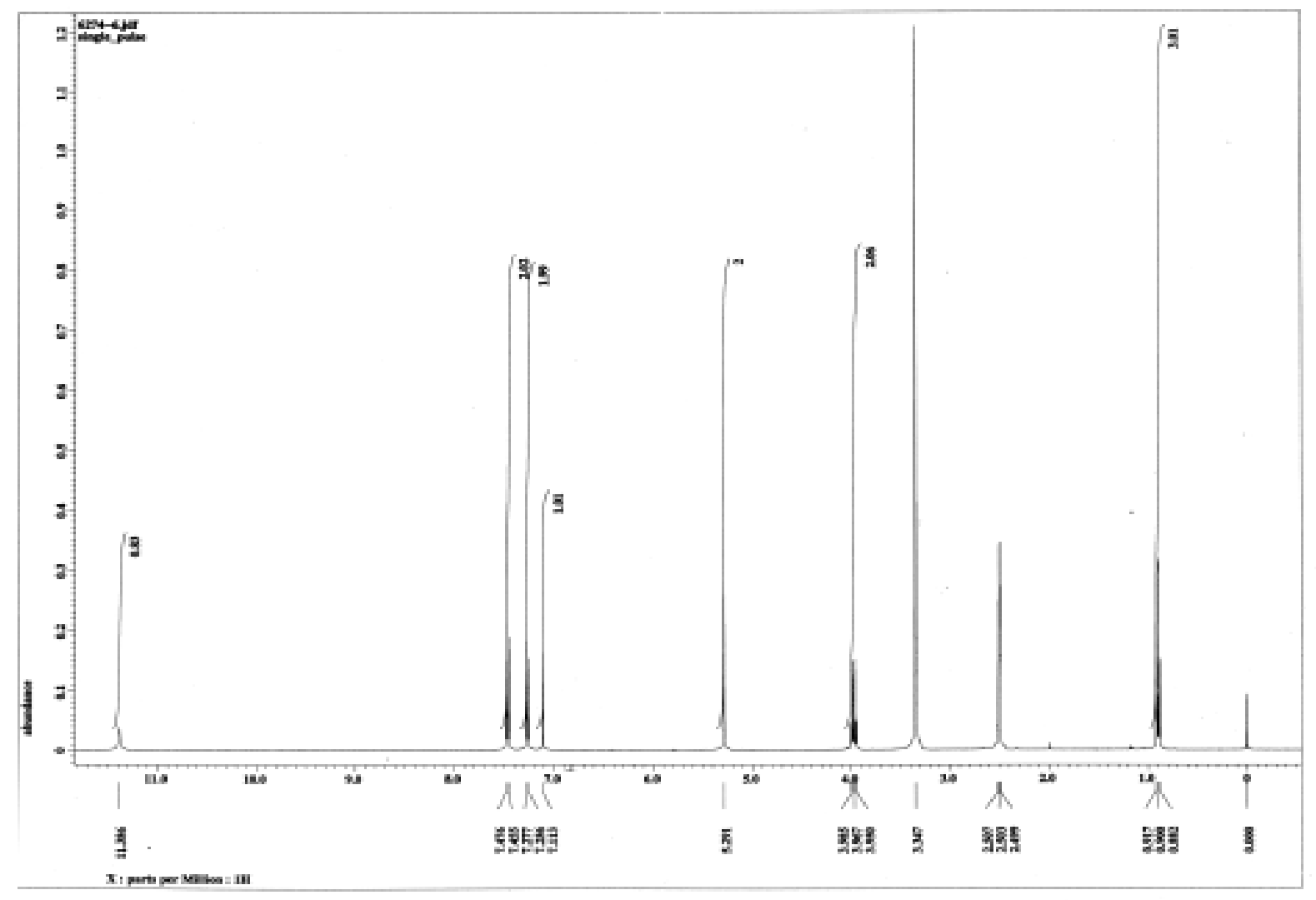

IR

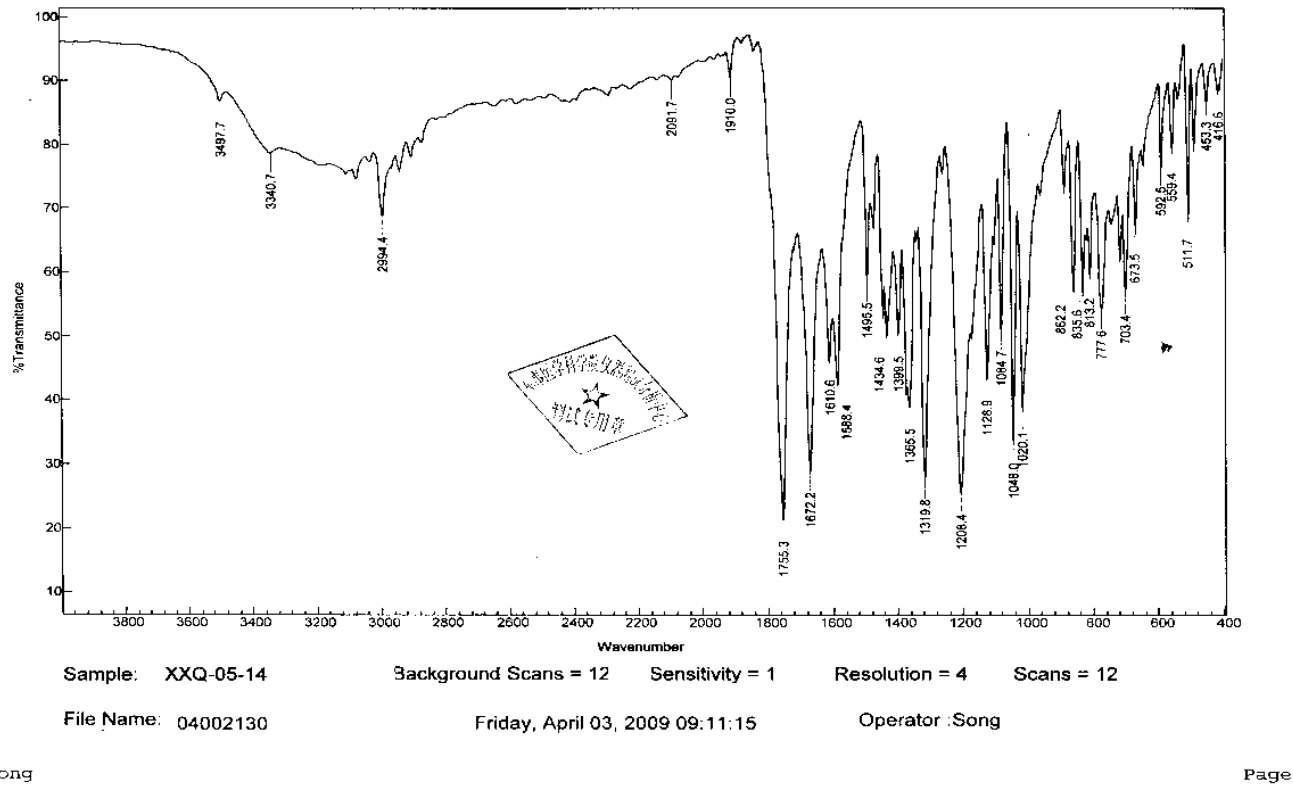

MS 


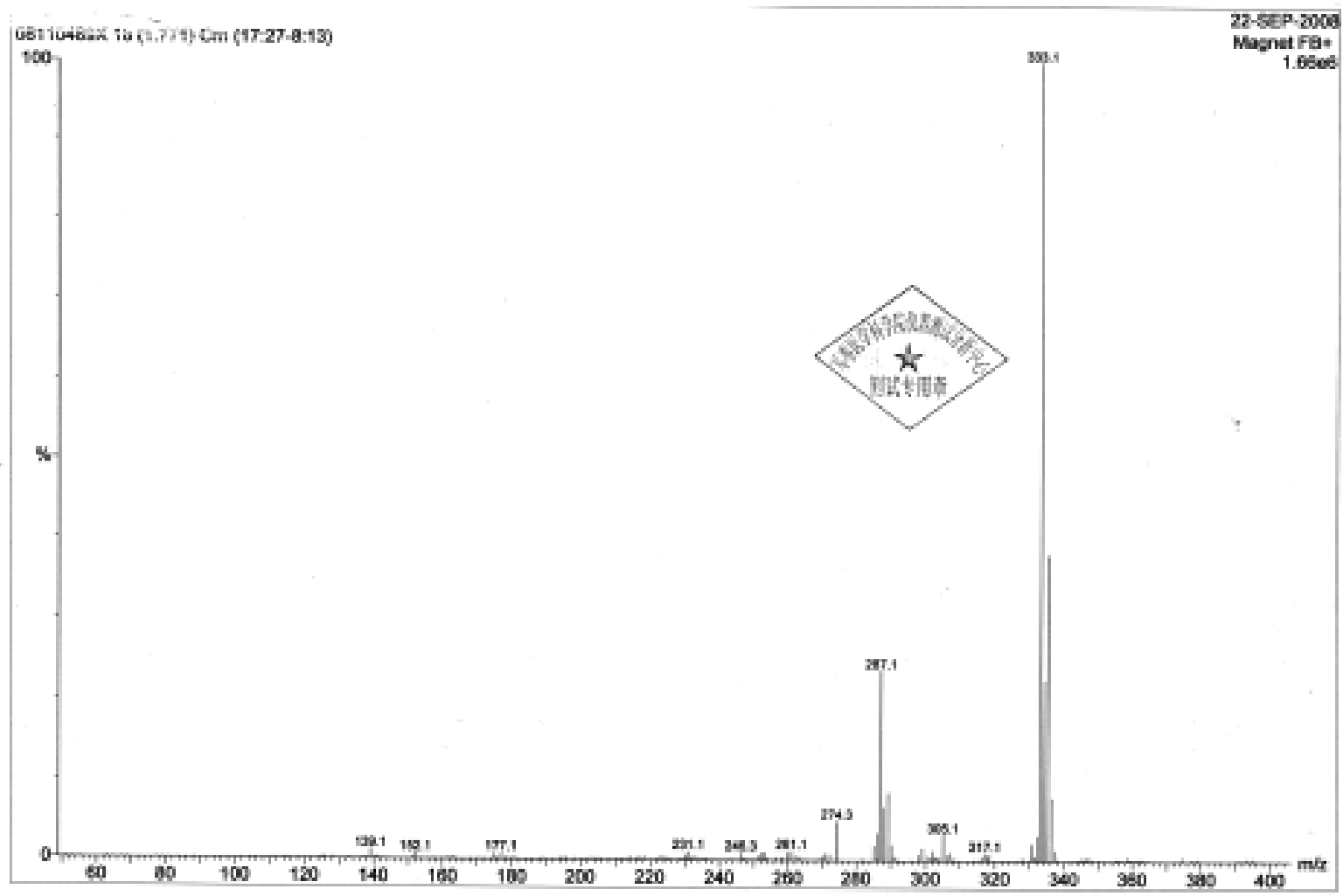

Compound 1e

${ }^{1} \mathrm{H}$ NMR

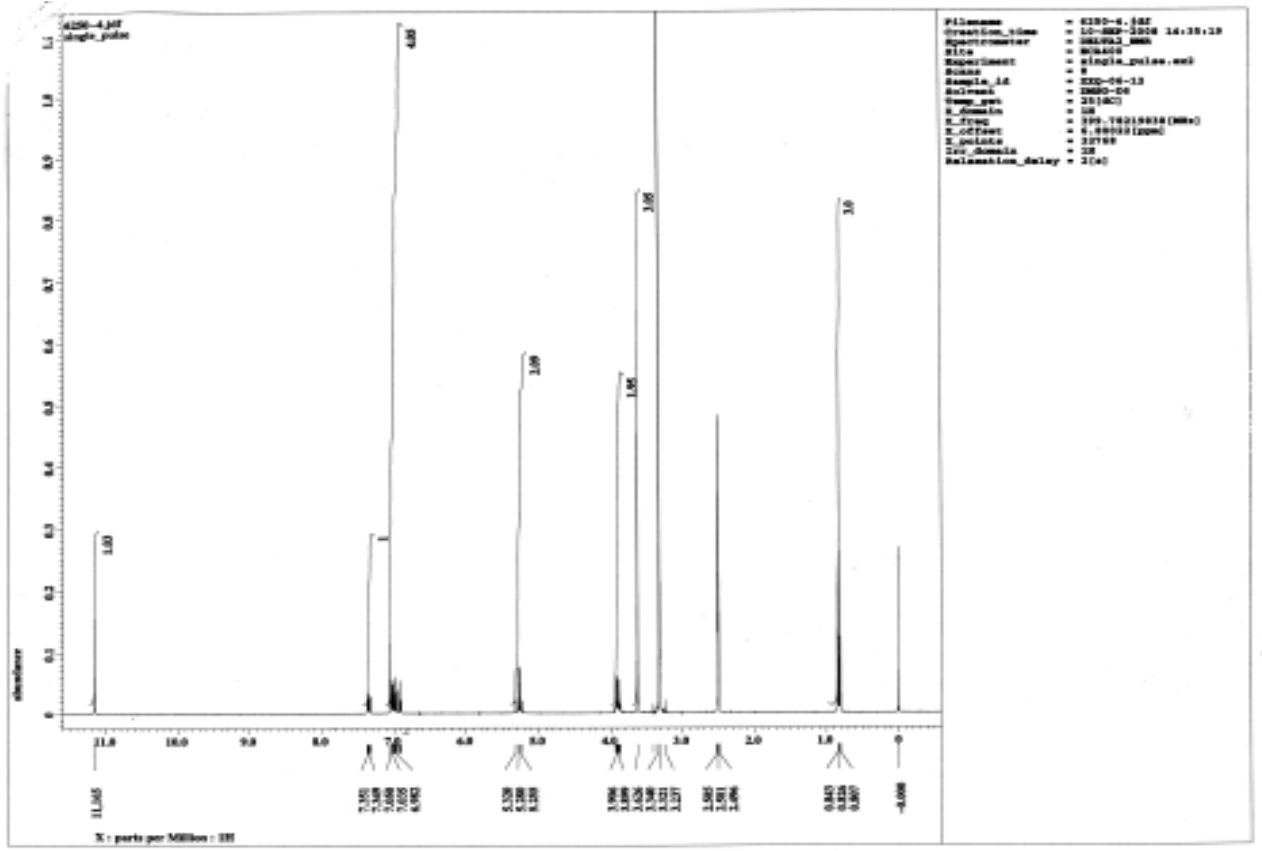

IR 


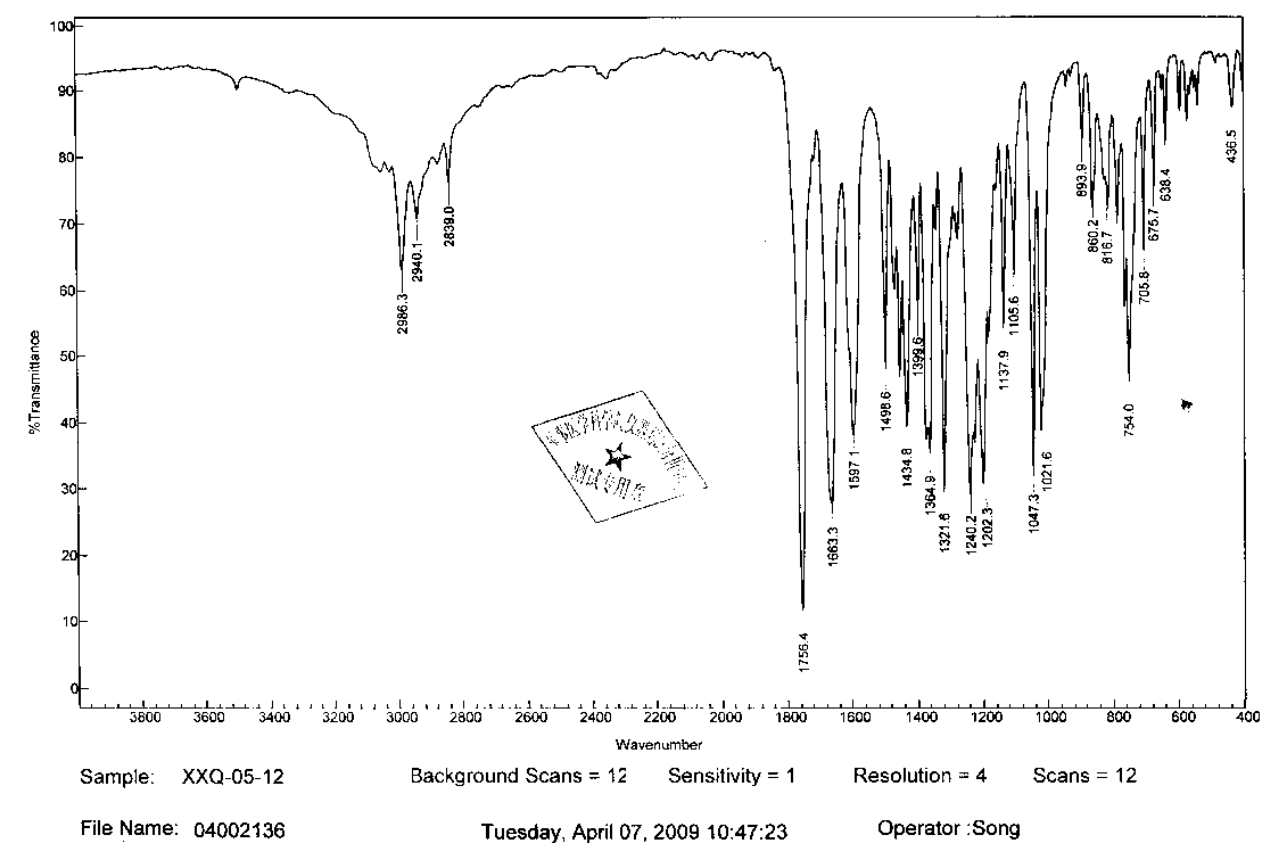

\section{MS}

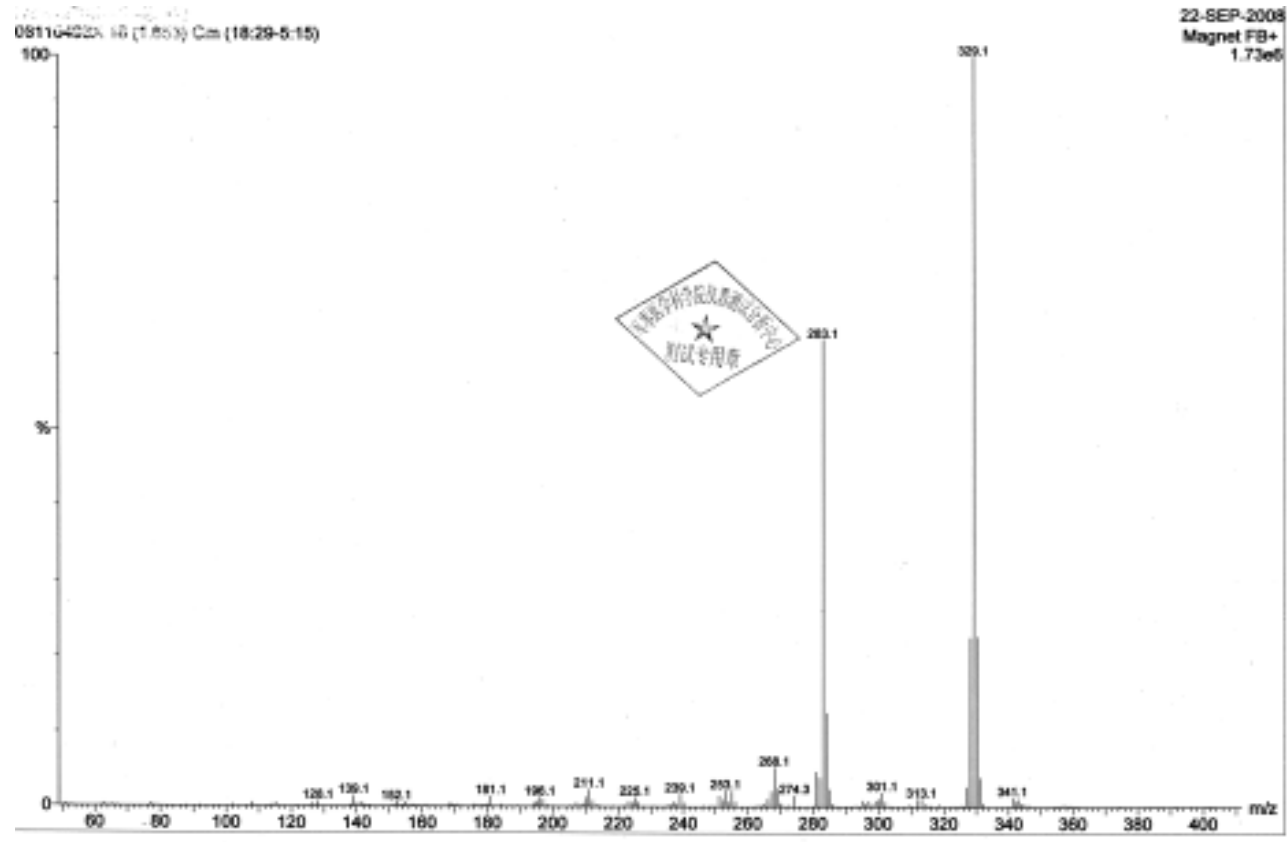

Compound $1 \mathrm{f}$ 
${ }^{1} \mathrm{H}$ NMR

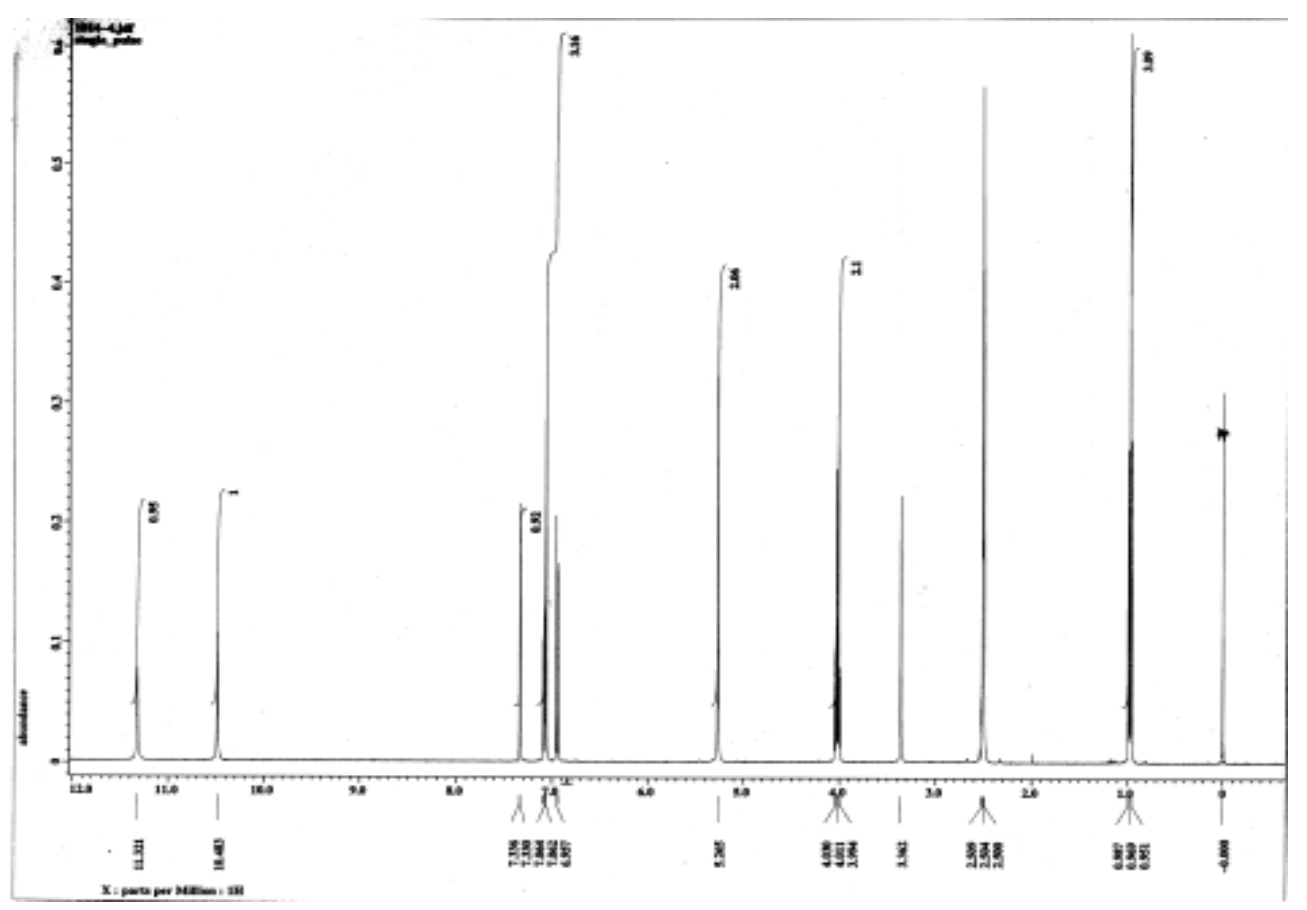

IR

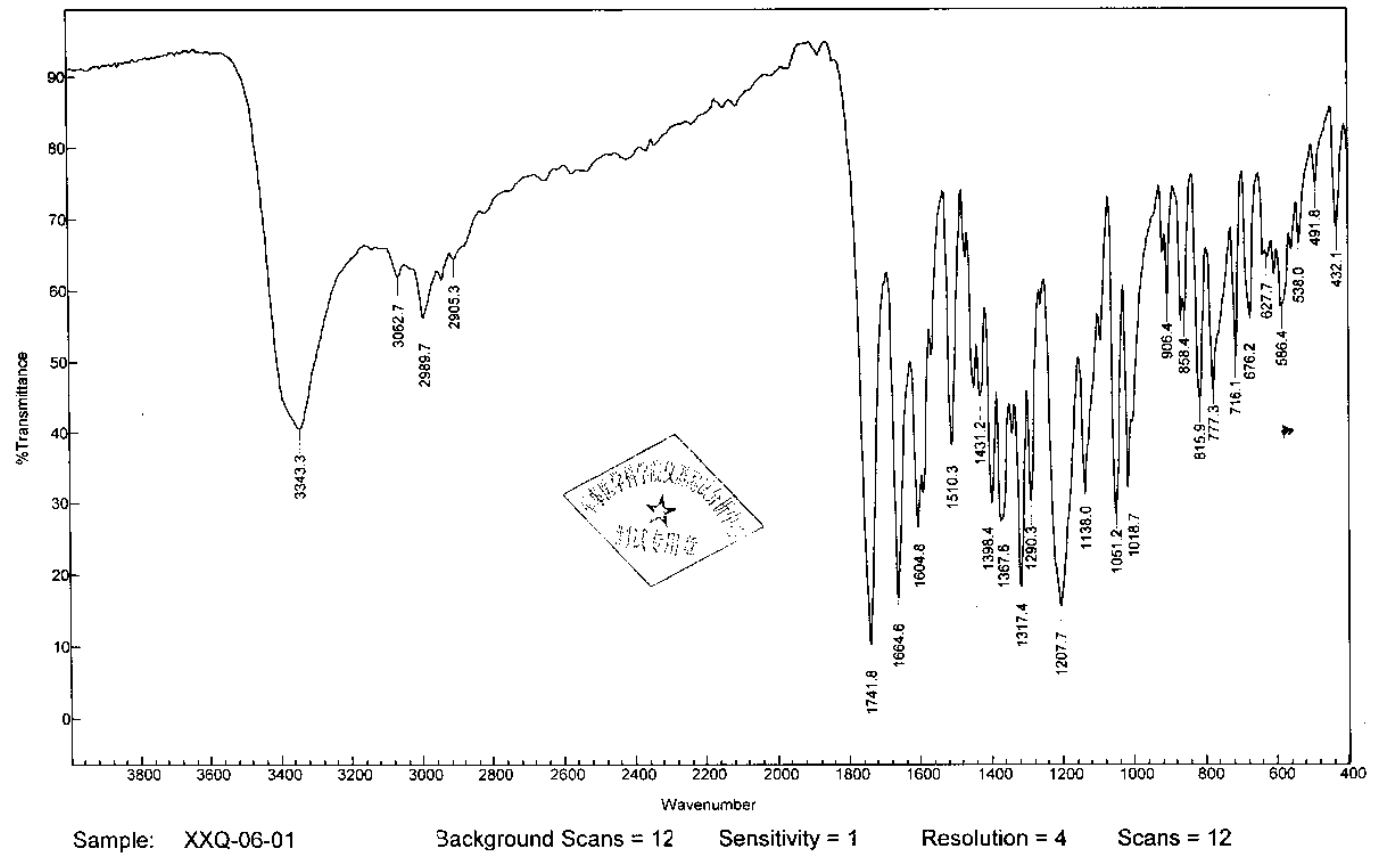




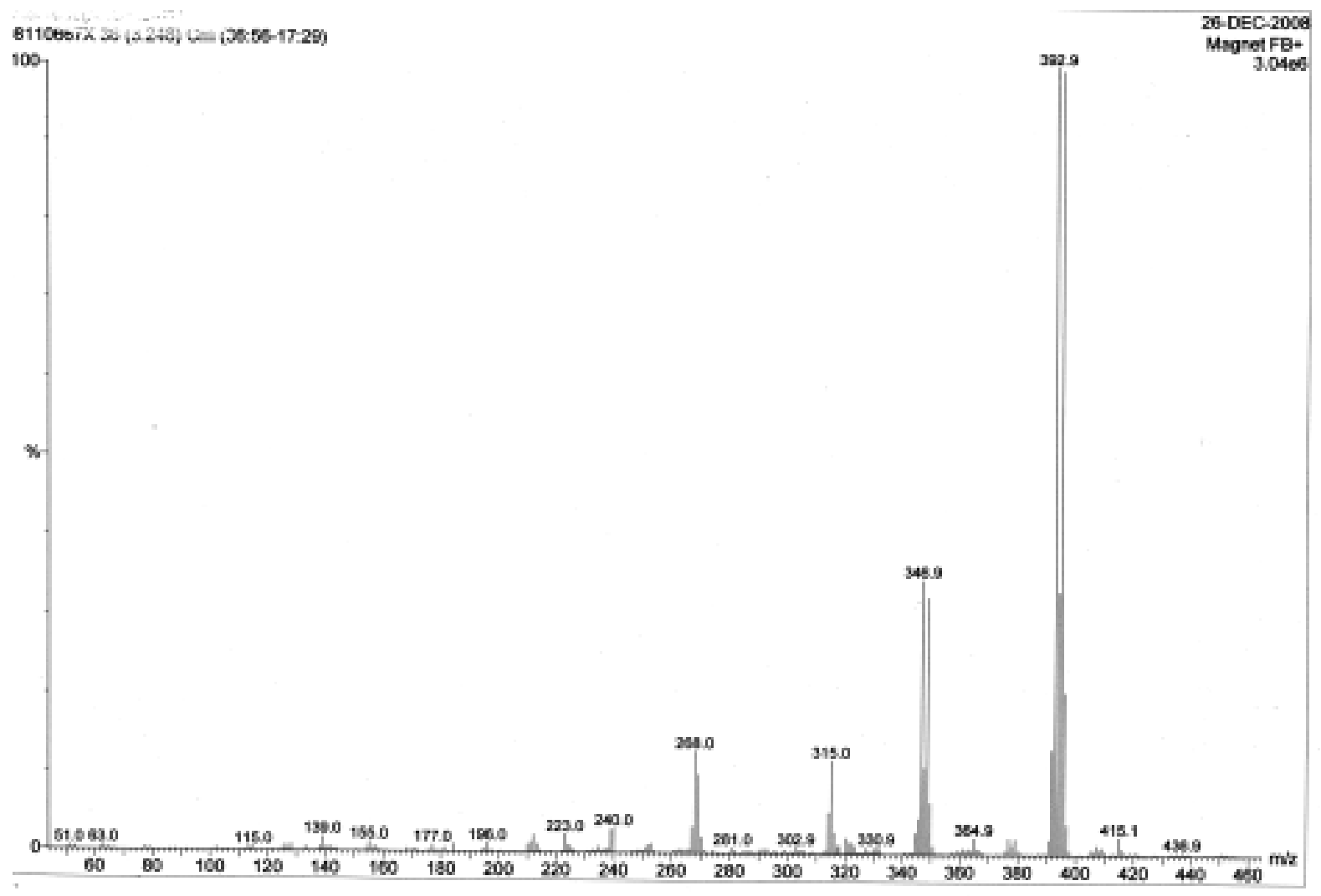

Compound $1 \mathrm{~g}$

${ }^{1} \mathrm{H}$ NMR

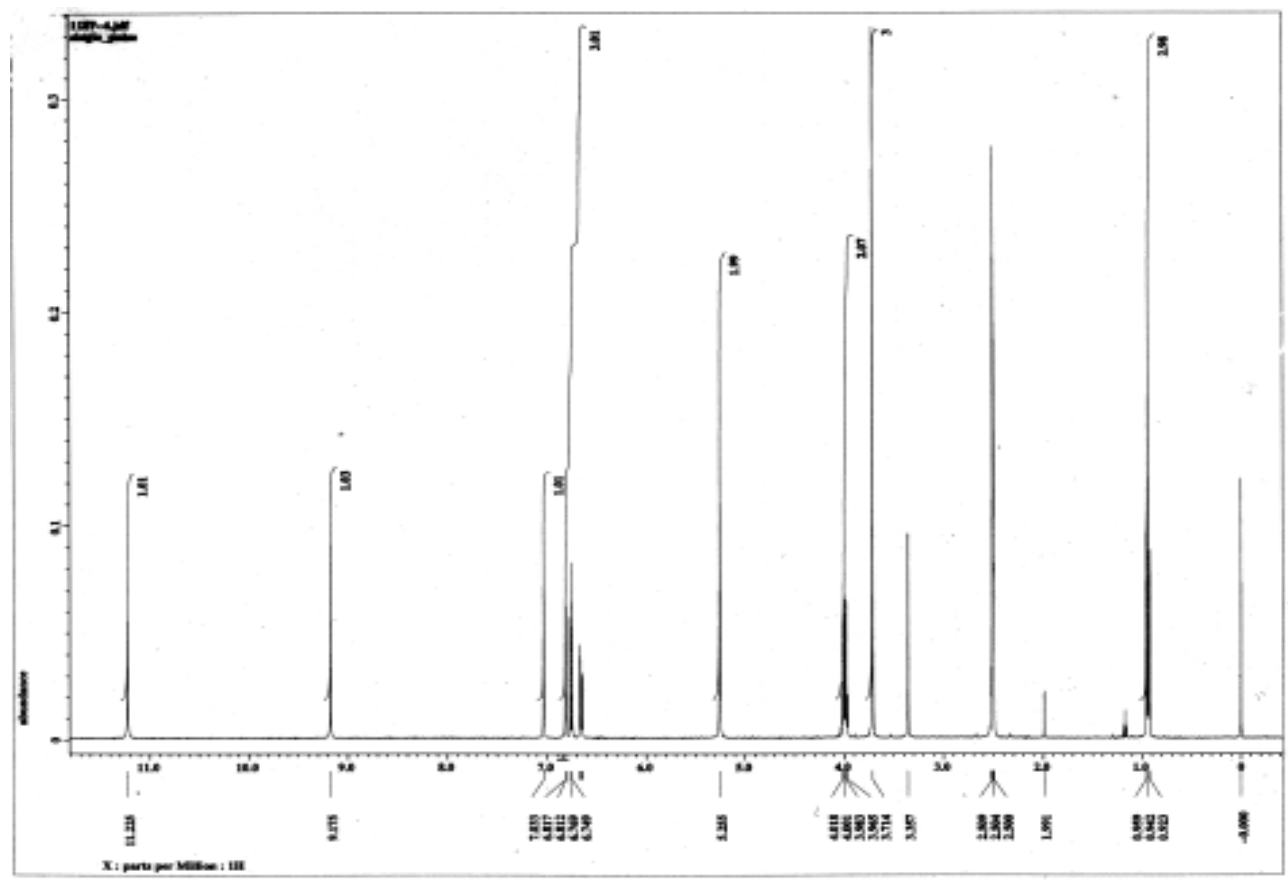

IR 


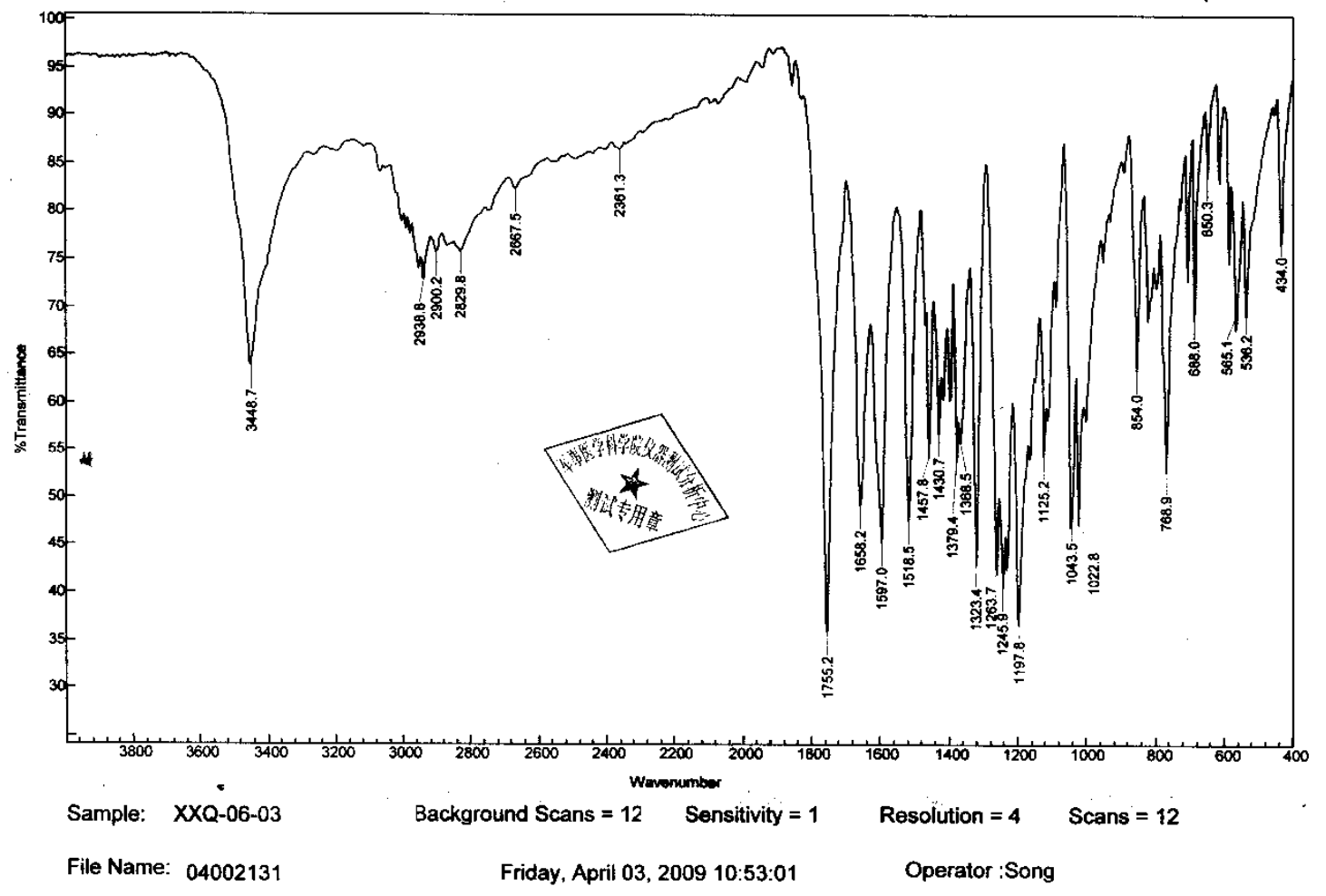

MS

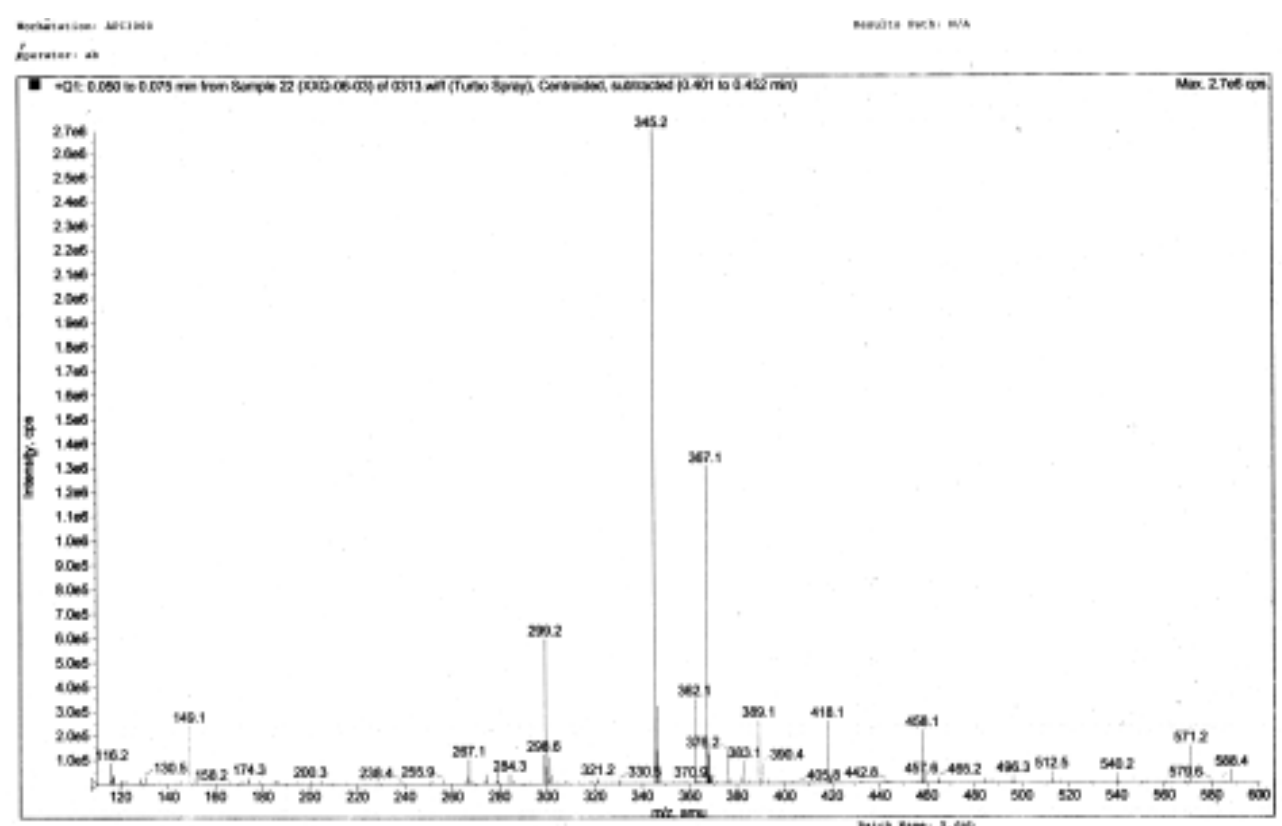

Compound $1 \mathrm{~h}$ 
${ }^{1} \mathrm{H}$ NMR

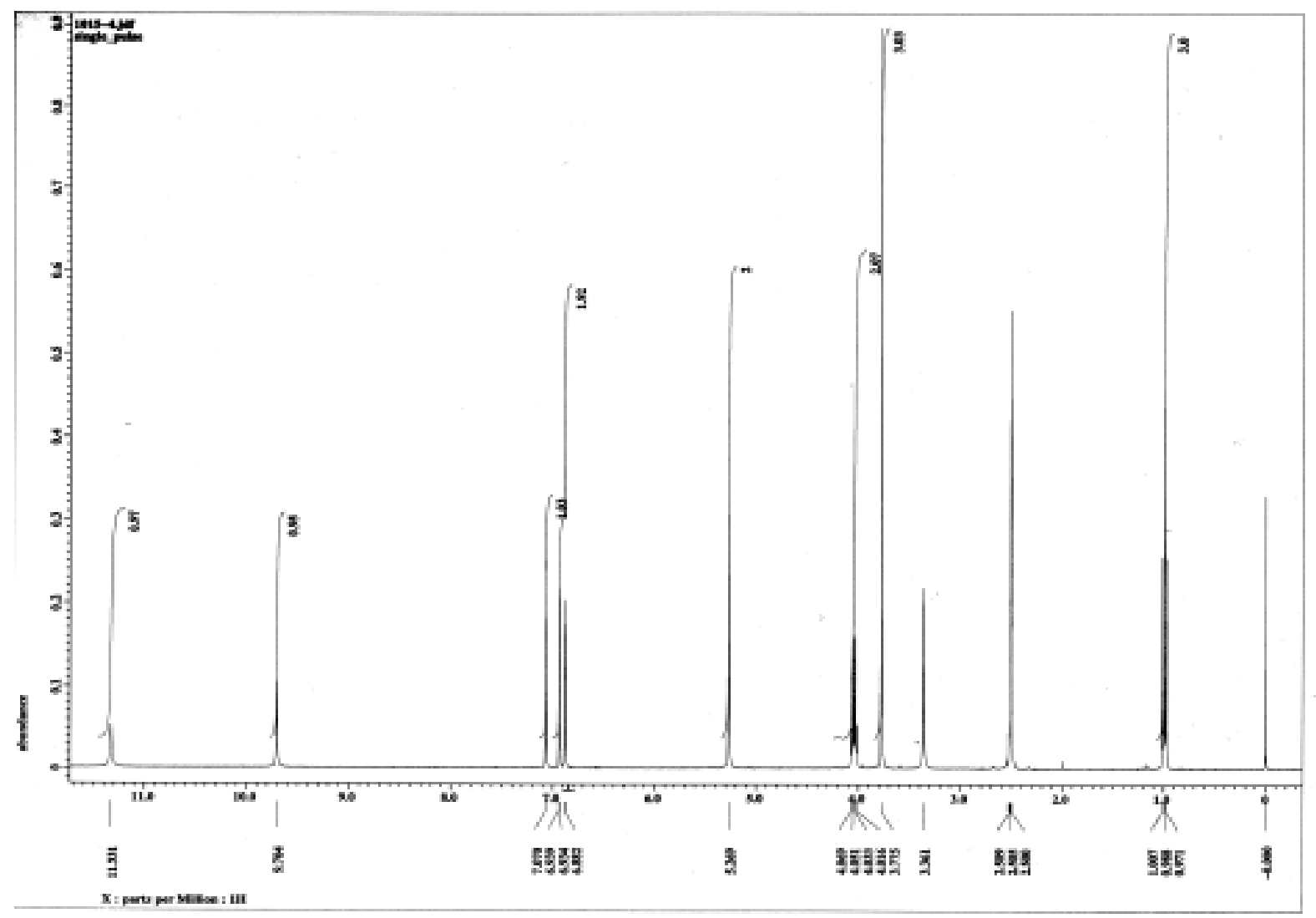

IR

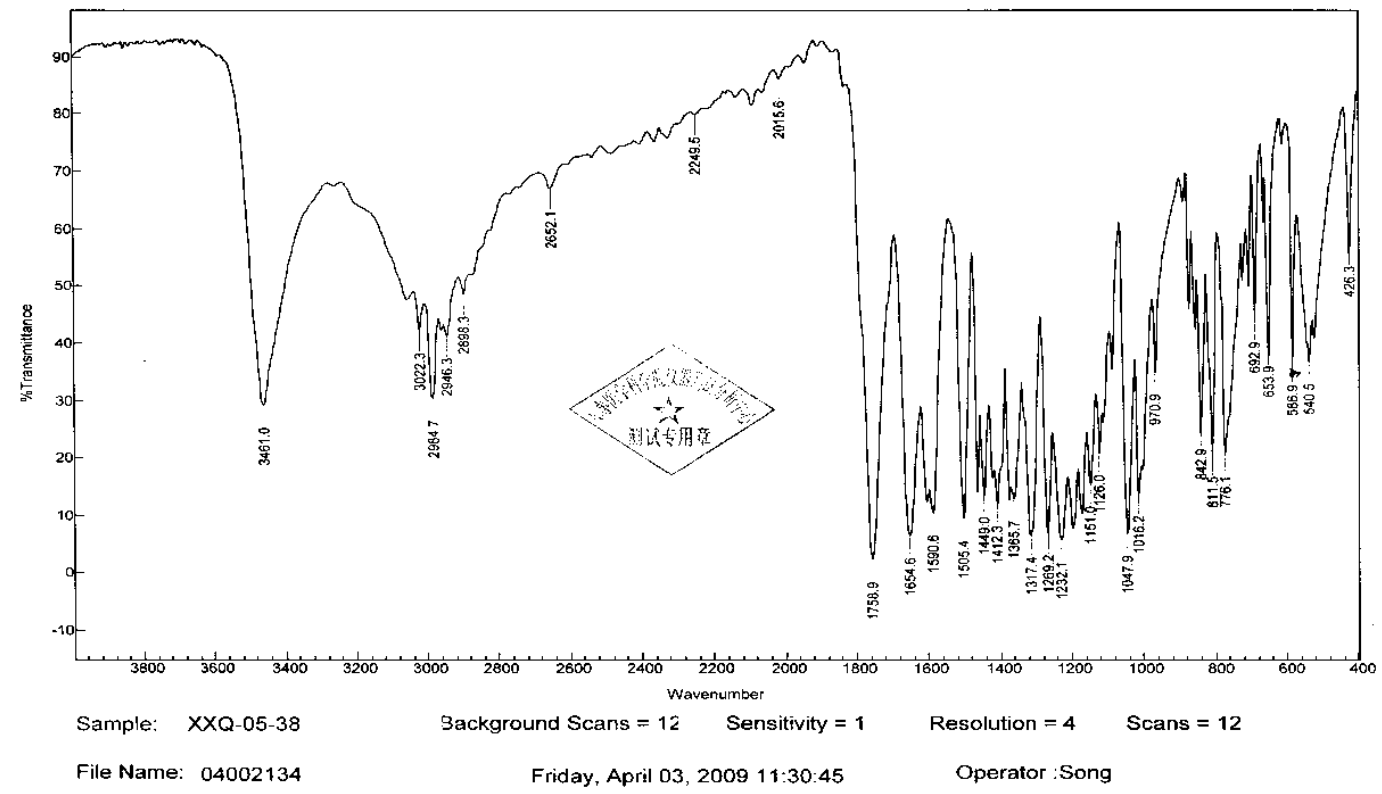




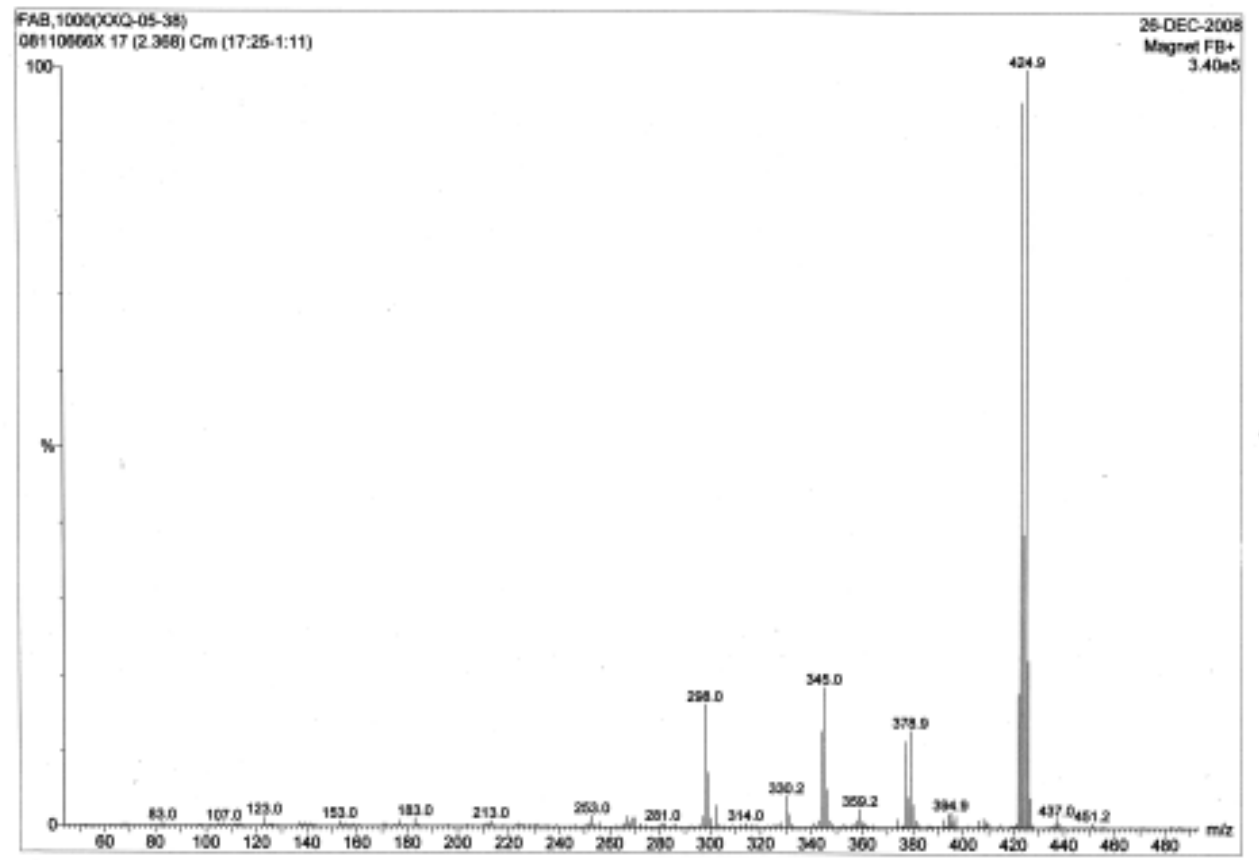

Compound $1 \mathrm{i}$

${ }^{1} \mathrm{H}$ NMR

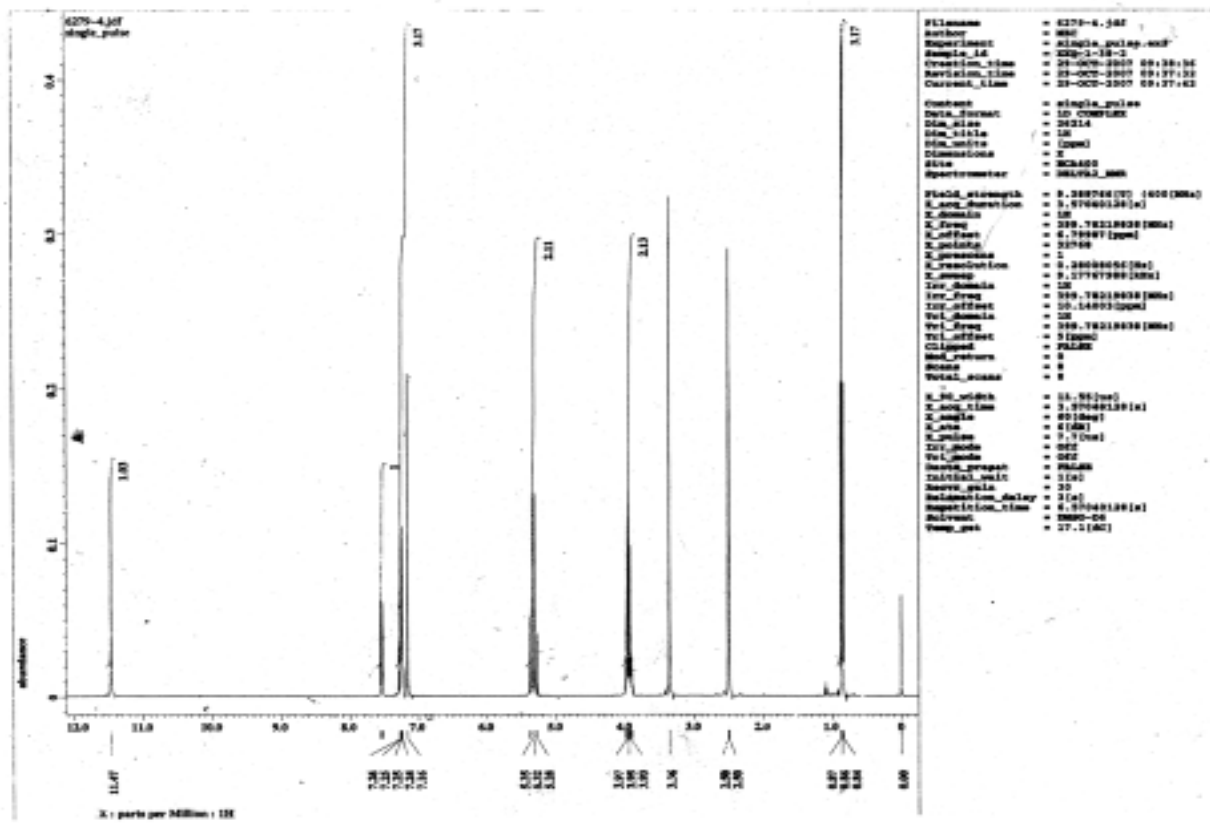

${ }^{13} \mathrm{C}$ NMR 


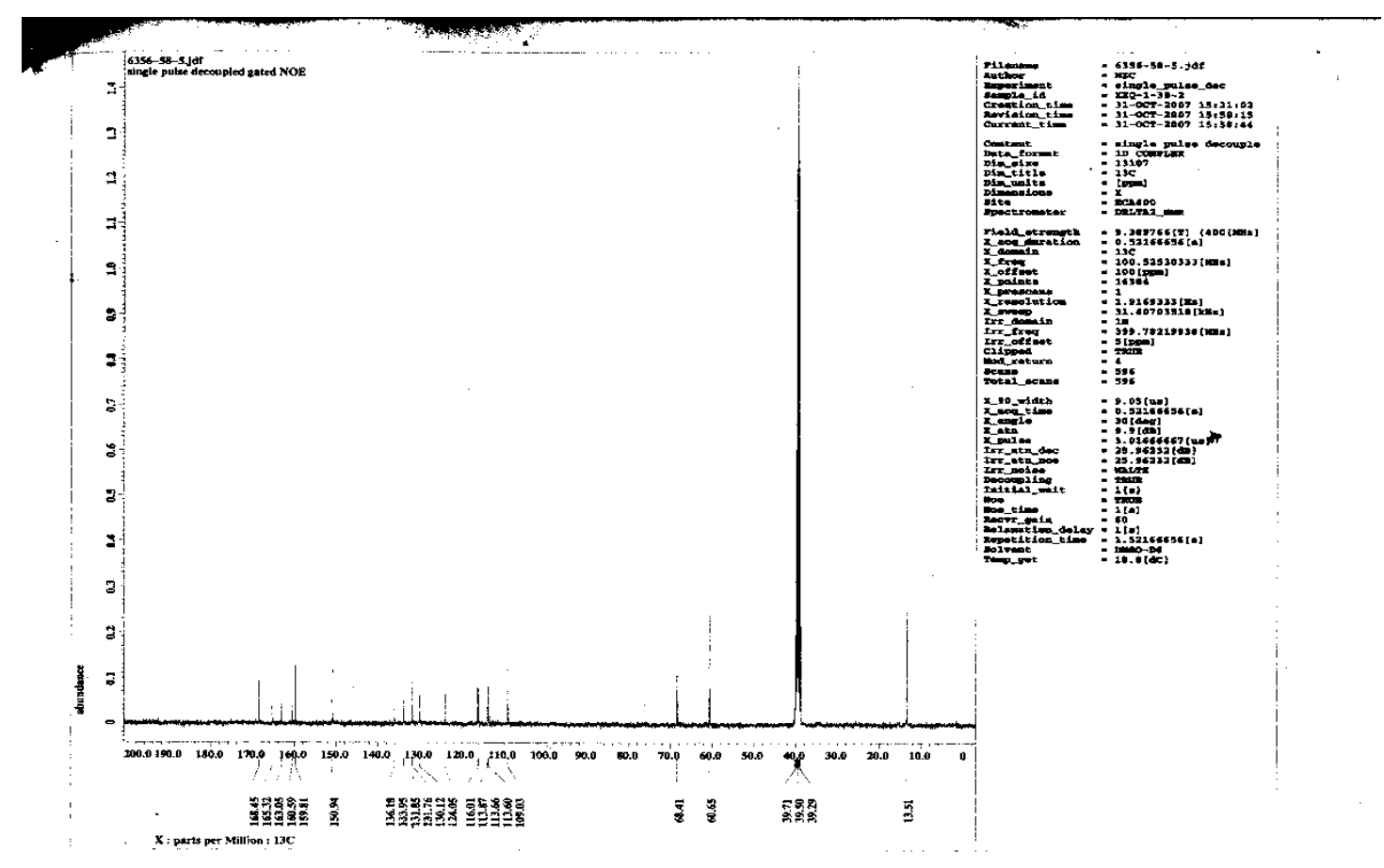

\section{HRMS}

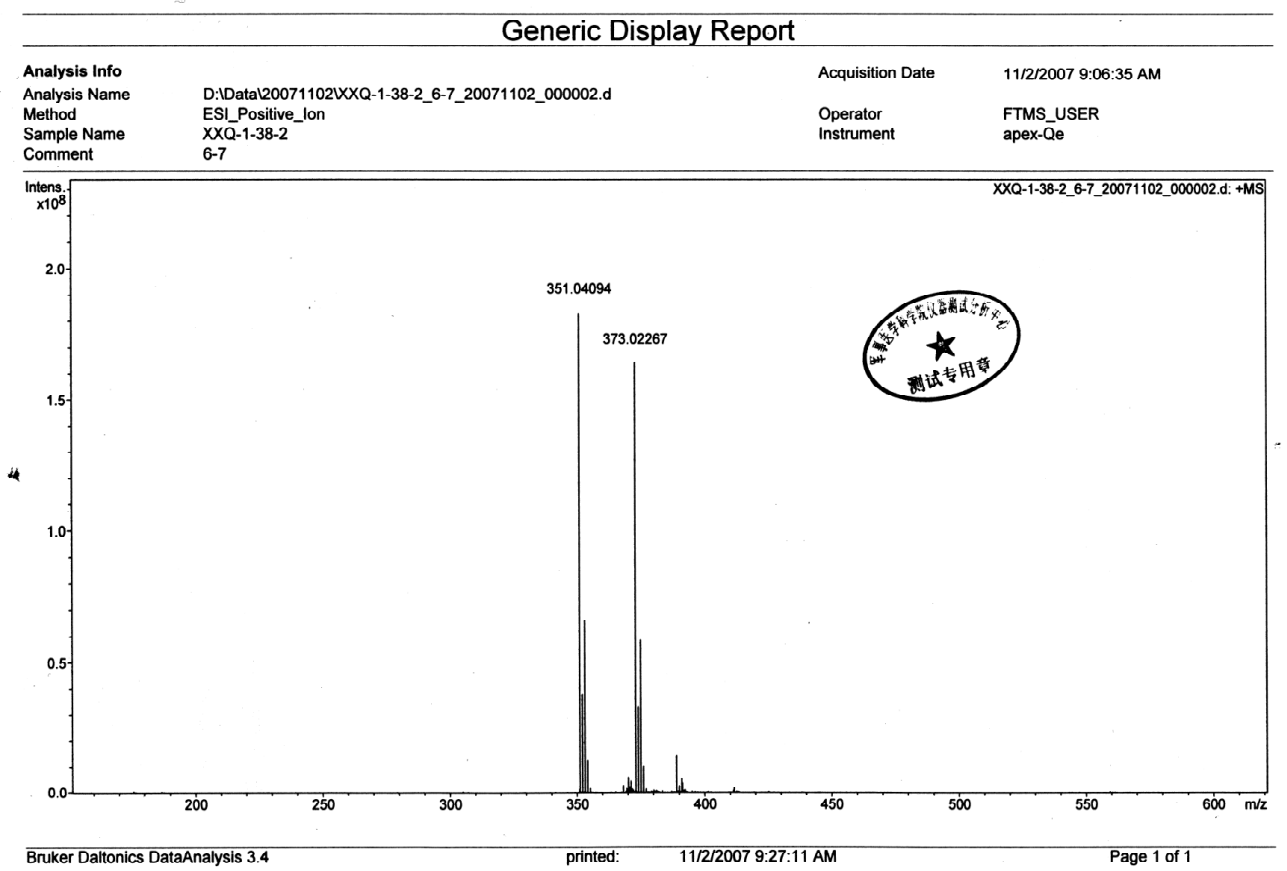

Compound $1 \mathrm{j}$

${ }^{1} \mathrm{H}$ NMR 


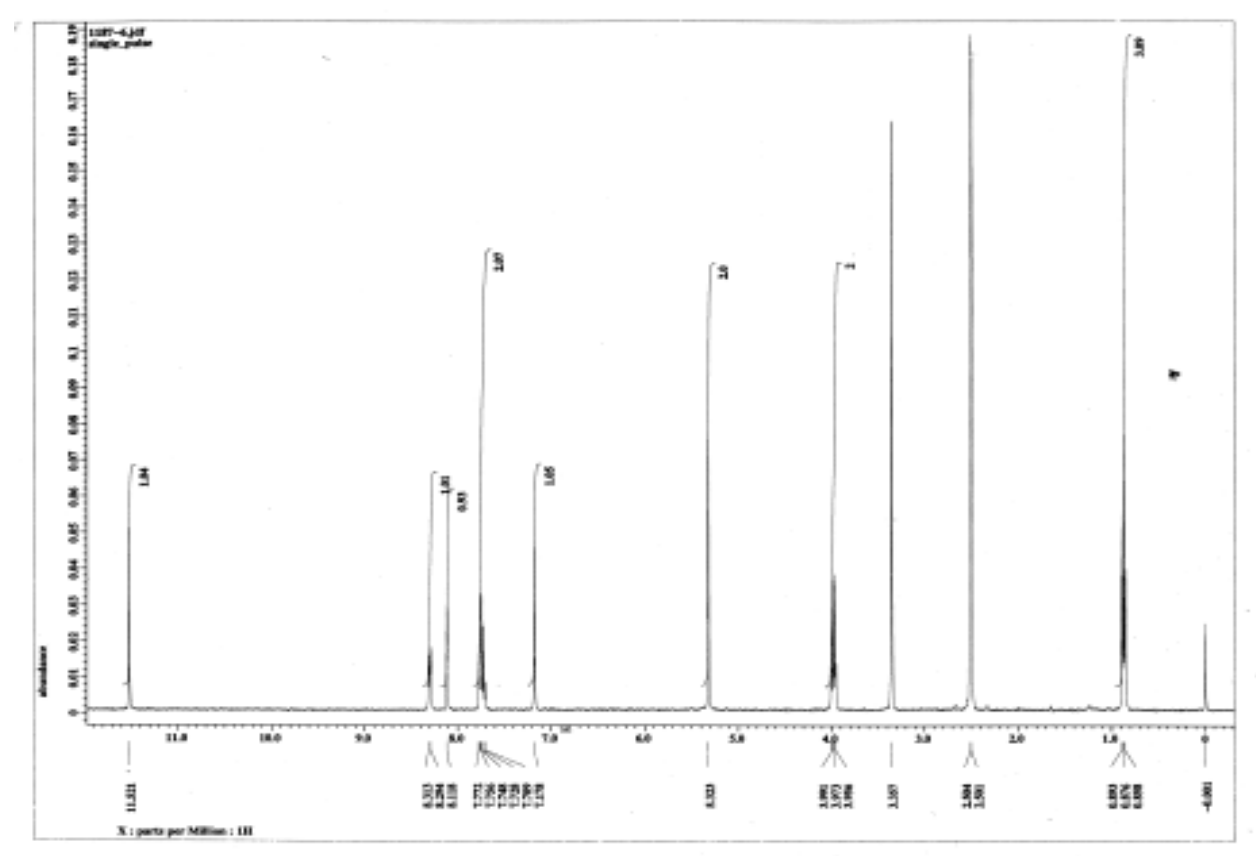

IR

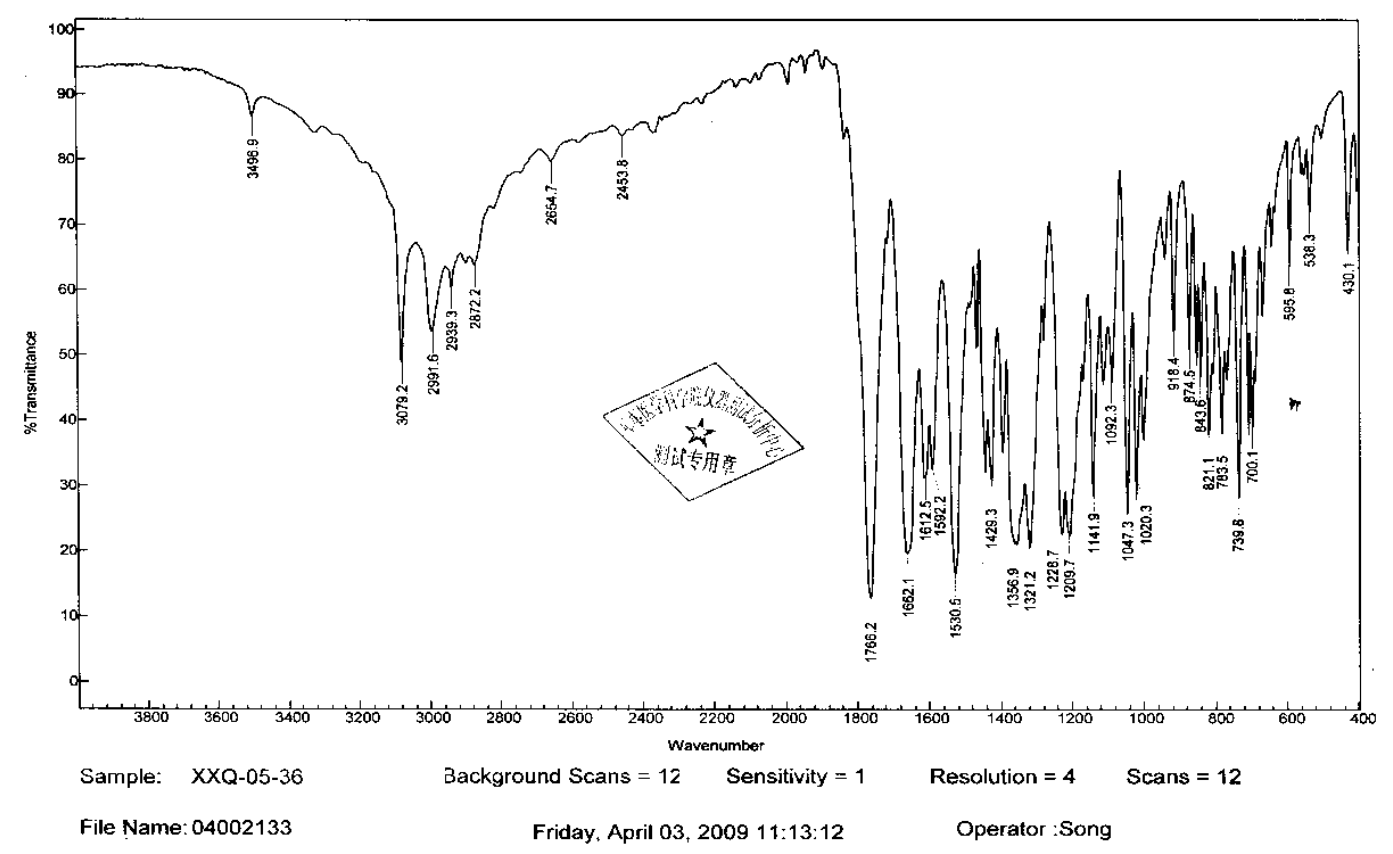




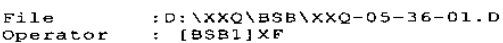

Acquired : 18 Dec 2008 14:55

8 Dec 2008

Instrument:

vial Number:

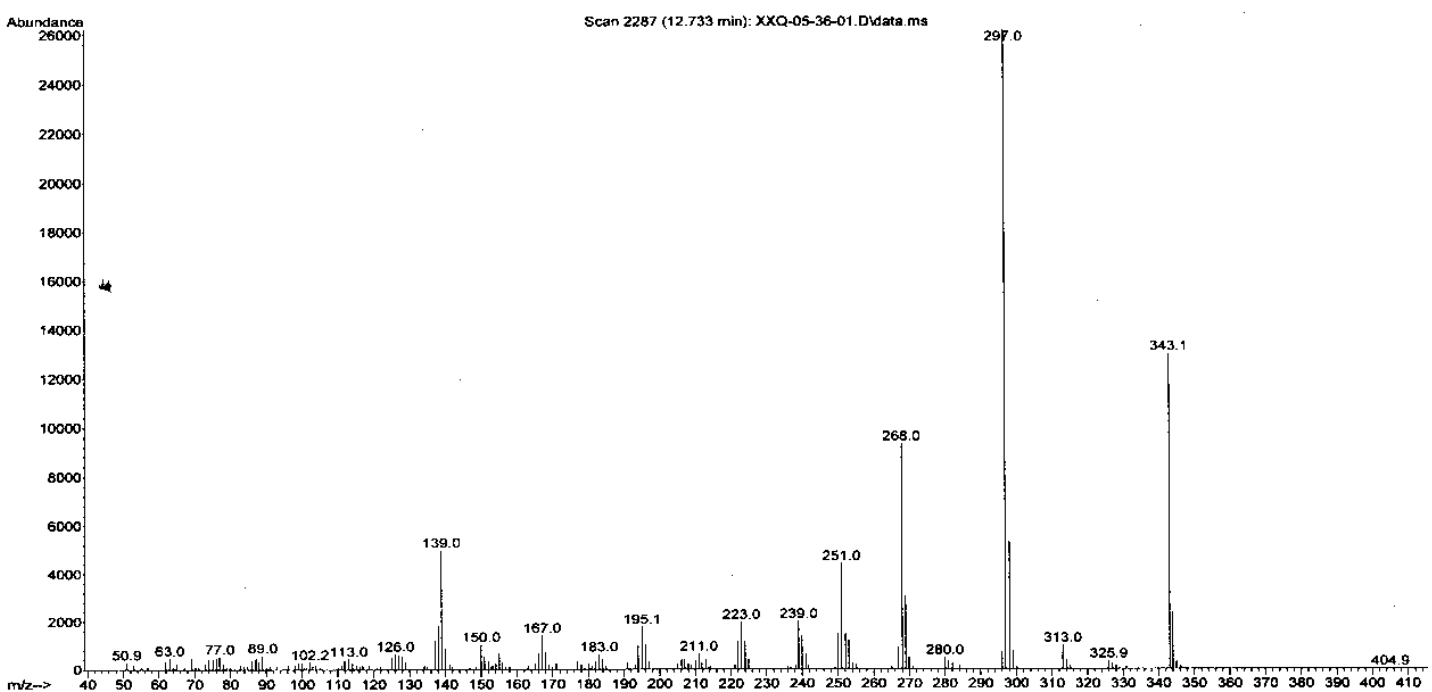

Element analysis $\quad(1 \mathrm{c}, 1 \mathrm{~d}, 1 \mathrm{e}, 1 \mathrm{f}, 1 \mathrm{~g}, 1 \mathrm{~h}, 1 \mathrm{j})$ 


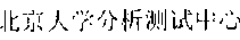

微招任机元素分析报告

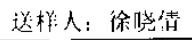

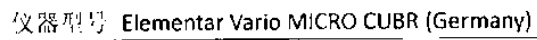

\begin{tabular}{|c|c|c|c|c|c|}
\hline \multirow{2}{*}{ 样品軓 } & \multicolumn{5}{|c|}{ 尚分舍至 $(\%)$} \\
\hline & $N$ & c & $\mathbf{H}$ & $S$ & 0 \\
\hline $1 c$ & & 54.13 & 3.56 & & \\
\hline $1 \mathrm{c}$ & & 54.08 & 3.53 & & \\
\hline $1 d$ & & 51.38 & 3.98 & & \\
\hline 1d & & 61.35 & 3.99 & & \\
\hline $1 e$ & & 65.55 & 4.95 & & \\
\hline $1 f$ & & 52.01 & 3.38 & & \\
\hline 1f & & 51.95 & 3.37 & & \\
\hline $1 \mathrm{~g}$ & & 62.42 & 4.71 & & \\
\hline $1 g$ & & 62.41 & 4.76 & & \\
\hline $1 \mathrm{~h}$ & & 51.16 & 3.62 & & \\
\hline ith & & 51.23 & 3.63 & & \\
\hline $1 j$ & 4.12 & 59.56 & 3.92 & & \\
\hline $1 j$ & 4.08 & 59.51 & 3.87 & & \\
\hline
\end{tabular}

